\title{
SMOOTHNESS ANALYSIS OF SUBDIVISION SCHEMES BY PROXIMITY
}

\author{
JOHANNES WALLNER
}

\begin{abstract}
Linear curve subdivision schemes may be perturbed in various ways, e.g. by modifying them such as to work in a manifold, surface, or group. The analysis of such perturbed and often nonlinear schemes " $T$ " is based on their proximity to the linear schemes " $S$ " which they are derived from. This paper considers two aspects of this problem: One is to find proximity inequalities which together with $C^{k}$ smoothness of $S$ imply $C^{k}$ smoothness of $T$. The other is to verify these proximity inequalities for several ways to construct the nonlinear scheme $T$ analogous to the linear scheme $S$. The first question is treated for general $k$, whereas the second one is treated only in the case $k=2$. The main result of the paper is that convergent geodesic / projection / Lie group analogues of a certain class of factorizable linear schemes have $C^{2}$ limit curves.
\end{abstract}

\section{INTRODUCTION}

Curve subdivision schemes in general consist of repeated refinement of control polygons. Especially well studied are the linear schemes with rules for defining the control points at the finer level as finite linear combinations of control points in the coarser level — see e.g. [7], [24], and [12].

This paper is a sequel to [22], which defines a wide class of curve subdivision schemes on manifolds, and analyzes convergence and $C^{1}$ smoothness. The analysis of such a nonlinear scheme is performed by its proximity to the corresponding linear scheme from which it was derived. In that sense the nonlinear scheme is a certain perturbation of the linear one. We give upper bounds on the magnitude of the perturbation which are sufficient for $C^{k}$ smoothness of the limit curves of a nonlinear scheme, if the original linear scheme has the same property.

As a main example for such a perturbation we consider the schemes " $T$ " defined in surfaces, Lie groups, and Riemannian manifolds, which are analogous to curve schemes " $S$ " defined in a vector space. Convergence and $C^{1}$ smoothness of these scheme are treated in [22]. The second part of the present paper deals with a certain class of factorizable linear schemes, where we can show that $S$ and $T$ are close enough for $T$ to produce $C^{2}$ limit curves, for all control polygons for which the subdivision process converges.

2000 Mathematics Subject Classification. 41A25,53B20,68U05,26A24,22E05.

Key words and phrases. nonlinear subdivision, smoothness, geodesics. 
Perturbations introduced by discretization and imcomplete computation via iterative algorithms are studied in [23] together with Computer graphics applications.

For an overview on previous work on nonlinear subdivision schemes and proximity, we refer the reader to the introduction of [22]. Here we only mention that analysis by proximity to a linear scheme is a technique which was used before in various situations, such as in the papers [11] and [4]. Smoothness analysis of other nonlinear subdivision schemes which occur in the literature (e.g. [25] and [20]) is mainly along different lines.

The outline of this paper is as follows: After introducing notation and presenting basic facts concerning linear schemes, Section 2 proceeds with the smoothness analysis of nonlinear schemes. We introduce convergence, smoothness, and proximity conditions, which generalize those of [22]. We show that in case that proximity of order $k-1$ holds for a linear scheme $S$ and a nonlinear scheme $T$, we can prove $C^{k}$ smoothness of limit curves generated by $T$, for a wide class of $C^{k}$ linear schemes $S$. Section 3 briefly discusses special cases of proximity conditions. A major part of the paper is Section 4, where we show that a certain class of nonlinear curve subdivision schemes and their analogous linear schemes fulfill a first order proximity condition. Section 5 combines results of previous sections and concludes $C^{2}$ smoothness for certain projection schemes, and certain geodesic schemes in surfaces, Riemannian manifolds, and Lie groups.

\section{Convergence And Smoothness Analysis}

2.1. Notation and basics on linear schemes. We consider sequences $p=$ $\left(p_{i}\right)_{i \in \mathbb{Z}}$ of points, which are also referred to as polygons. A subdivision scheme $S$ is a mapping which takes a point sequence $p$ as input, and which has another point sequence $S p$ as output. We assume that there is an integer dilation factor $N$ such that for all polygons $p, q$ the relation $q_{i}=p_{i+1}$ for all $i$ implies that $(S q)_{i}=(S p)_{i+N}$.

We focus on subdivision schemes whose definition uses the notion of average or affine combination. We shall presently see that this is not a restriction. We use the notation

$$
\operatorname{av}_{\alpha}(x, y):=(1-\alpha) x+\alpha y .
$$

For instance, degree $n$ B-spline subdivision " $S_{(n)}$ " according to [14] has $N=2$ and is recursively defined by one splitting step and $n$ averaging steps:

$$
\begin{aligned}
& \left(S_{(0)} p\right)_{2 i}=\left(S_{(0)} p\right)_{2 i+1}=p_{i}, \\
& \left(S_{(m)} p\right)_{i}=\operatorname{av}_{1 / 2}\left(\left(S_{(m-1)} p\right)_{i},\left(S_{(m-1)} p\right)_{i+1}\right), \quad m=1, \ldots, n .
\end{aligned}
$$

For a linear scheme there exists a sequence $a=\left(a_{i}\right)_{i \in \mathbb{Z}}$ such that

$$
S p_{j}=\sum_{i} a_{j-N i} p_{i}
$$


$a$ is called the mask of $S$, and is said to be finite if only finitely many $a_{i}$ 's are nonzero. The subdivision scheme is affinely invariant, if

$$
\sum_{i} a_{j-N i}=1, \quad j=0, \ldots, N-1 .
$$

Any convergent curve subdivision scheme is affinely invariant (cf. [7]). It is not difficult to show (cf. [22]) that the rules of the scheme can be expressed in terms of repeated affine averages:

Theorem 1. Any affinely invariant linear subdivision rule $S$ with finite mask is expressible via the "av" operator.

This representation of affinely invariant linear subdivision schemes, which is not unique, will be used to define nonlinear schemes on manifolds analogous to linear schemes (see Section 4). An explicit example is furnished by (2.2).

2.2. The limit of a subdivision scheme. For a polygon $p$, we use the notation $\Delta p$ for the sequence of differences: $\Delta p_{i}=p_{i+1}-p_{i}$. Further we define

$$
\|p\|_{\infty}=\sup _{i}\left\|p_{i}\right\|, \quad d(p)=\|\Delta p\|_{\infty} .
$$

The limit of a sequence of polygons which are getting denser and denser is best dealt with via the limit of a sequence of functions: for a sequence $p$ of points, define $\mathcal{F}_{j}(p)$ to be the piecewise linear function which is linear in the intervals $\left[i N^{-j},(i+1) N^{-j}\right](i \in \mathbb{Z})$, and which has the property that $\mathcal{F}_{j}(p)\left(i N^{-j}\right)=p_{i}$. If $T$ is a subdivision scheme, we use the notation

$$
f=\mathcal{F}_{0}(p), \quad T f=\mathcal{F}_{1}(T p), \quad T^{2} f=\mathcal{F}_{2}\left(T^{2} p\right), \ldots
$$

Then

$$
T^{\infty} f=\lim _{j \rightarrow \infty} T^{j} f
$$

is the limit curve, which is also denoted by $T^{\infty} p$. It is obvious from construction that

$$
\|p-q\|_{\infty}=\left\|\mathcal{F}_{j}(p)-\mathcal{F}_{j}(q)\right\|_{\infty} .
$$

2.3. Convergence and smoothness conditions. This subsection introduces conditions called 'convergence' and 'smoothness' conditions. It will be seen later that indeed they are the main ingredients in our proofs concerning the convergence of a nonlinear subdivision scheme, and the continuity and smoothness of its limit curves.

The 'mixed' condition in Def. 2 below uses polynomials $P(x)$ with the property that $P(n) \geq 0$ for all nonnegative integers $n$. We call such polynomials nonnegative for short. It is obvious that sum, positive multiples, and products of nonnegative polynomials are again nonnegative polynomials, and so is the summation polynomial $R$ of a nonnegative polynomial $P$, which is defined by $R(n)=\sum_{i=0}^{n} P(i)$. 
Definition 1. A subdivision scheme $S$ is said to satisfy a convergence condition with factor $\mu_{0}<1$, if

$$
d\left(S^{l} p\right) \leq \mu_{0}^{l} d(p) \text { for all } l, p .
$$

Definition 2. A linear scheme $S$ with dilation factor $N$ is said to satisfy a smoothness condition of order $k$ with factors $\mu_{0}, \ldots, \mu_{k}<1$, if in addition to (2.9) for all $l, p$,

$$
d\left(N^{l j} \Delta^{j} S^{l} p\right) \leq \mu_{j}^{l} d\left(\Delta^{j} p\right), \quad j=1, \ldots, k .
$$

A not necessarily linear scheme $S$ with dilation factor $N$ is said to satisfy a mixed smoothness condition of order $k$, if (2.9) holds and there are $\mu_{1}, \ldots, \mu_{k}<1$, such that for all $l, p$

$$
d\left(N^{j l} \Delta^{j} S^{l} p\right) \leq \mu_{j}^{l} P_{j}(l) d(p), \quad j=1, \ldots, k,
$$

where $P_{j}$ is a nonnegative polynomial.

The smoothness condition of (2.10) is well known in the smoothness analysis of linear subdivision schemes. Mixed conditions of the type (2.11) appear in our smoothness analysis of nonlinear schemes below. In fact, a sequence dominated by $\mu^{l} P(l)$ for a polynomial $P$, is also eventually dominated by a constant times $\mu^{l+\epsilon}$, for any $\epsilon>0$. We thus could avoid using the "mixed conditions" altogether and work solely with the well known exponential decay of (2.10).

Most of our statements consider polygons $p$ whose points are contained in some subset $M$ of $\mathbb{R}^{n}$, and fulfill the condition $d(p)<\varepsilon$. Such a class of polygons is denoted by $\mathcal{P}_{M, \varepsilon}$. The statements employ a scheme " $S$ ", which is linear and whose properties are known, and another scheme " $T$ ", which is to be analyzed ( $S$ is to aid the analysis). We will encounter the situation that smoothness conditions are true only for $p \in \mathcal{P}_{M, \delta}$ for some $\delta>0$.

2.4. Smoothness of linear schemes. The smoothness conditions (2.9) and (2.10) guarantee the smoothness of a linear scheme $[7,12]$. In this subsection we show how to compute the factors $\mu_{i}$ for linear schemes. Following $[7,8]$, we use the concept of $k$-th derived scheme $S_{k}$ of a linear subdivision scheme $S$, which is recursively defined by the equations $S_{0}=S$ and $S_{i}(\Delta p)=N \Delta S_{i-1} p$, leading to $S_{i} \Delta^{i} p=N^{i} \Delta^{i} S p$. For the convenience of the reader, we repeat some definitions and results here. In the analysis of linear schemes, it is customary to define the formal Laurent series $a(z), p(z), S p(z)$, and $\Delta p(z)$ with coefficients taken from the sequences $a$ (the mask of the scheme), $p$ (the control polygon), $S p$ (the subdivided control polygon), and $\Delta p$ (the difference polygon), respectively. For example, we have $a(z)=\sum a_{i} z^{i}$. These are called the generating functions of the respective sequences, and $a(z)$ is called the symbol of $S$. We have $S p(z)=a(z) p\left(z^{N}\right)$ and $\Delta p(z)=(1-z) p(z) z^{-1}$. It follows immediately that the symbol $a^{[1]}(z)$ of the derived scheme $S_{1}$ equals $a^{[1]}(z)=a(z) N z^{N-1} /\left(1+\cdots+z^{N-1}\right)$. If (2.4) holds 
(i.e., $S$ is affinely invariant), then this division is possible without remainder (i.e., the derived scheme exists).

For any subdivision scheme $S, m$ rounds of subdivision yield yet another scheme, $S^{m}$. If $S$ has dilation factor $N$, then the dilation factor of $S^{m}$ equals $N^{m}$. It follows from the formulas above that the symbol $c(z)$ of $S^{m}$ is given by $c(z)=a(z) a\left(z^{N}\right) \cdots a\left(z^{N^{m-1}}\right)$. In this paper, the norm $\|S\|$ of $S$ is the sup-norm,

$$
\|S\|=\sup _{\|p\|_{\infty} \leq 1}\|S p\|_{\infty} .
$$

In the following we assume that the mask $a$ is of finite support, and we have

$$
\|S\|=\max _{j=1}^{N} \sum_{i}\left|a_{j-N i}\right|
$$

Knowledge of the norms of the derived schemes yields factors $\mu_{j}$ as required by (2.9) and (2.10): We use $d\left(\Delta^{j} p\right)=\left\|\Delta^{j+1} p\right\|_{\infty}$ and compute

$$
d\left(N^{j} \Delta^{j} S p\right)=\frac{1}{N}\left\|S_{j+1} \Delta^{j+1} p\right\|_{\infty} \leq \frac{1}{N}\left\|S_{j+1}\right\| d\left(\Delta^{j} p\right) .
$$

It follows that we may let

$$
\mu_{j}=\frac{1}{N}\left\|S_{j+1}\right\|
$$

The B-spline subdivision rule of degree $n$ according to (2.2) has $N=2$ and the symbol

$$
a(z)=(1+z)^{n+1} /(2 z)^{n}, \quad n \geq 0 .
$$

Its first derived scheme is the $(n-1)$-st degree B-spline scheme. Equations (2.13) and (2.15) show that convergence and smoothness conditions up to degree $n-1$ are fulfilled with factors $\mu_{i}=1 / 2$. It is not difficult to see that this is an optimal value. For the convenience of the reader, a proof is included below.

Lemma 1. For an affinely invariant scheme $S$, the decay rate $\mu_{j}$ of $(2.15)$ obeys $\mu_{j} \geq 1 / N$.

Proof. This is an immediate consequence of $\left\|S_{j+1}\right\| \geq 1$, which is shown in two steps: First, $\|S\| \geq 1$, because otherwise $S$ would be contractive and not affinely invariant. Second we show that, in general, $\left\|S_{1}\right\| \geq\|S\|$, which by iteration implies $\left\|S_{j+1}\right\| \geq\|S\| \geq 1$. The symbols $a(z)$ of $S$ and $a^{[1]}(z)$ of its derived scheme $S_{1}$ have the relation $a(z)=N^{-1} z^{1-N} \widetilde{a}(z)$ with $\widetilde{a}(z)=\left(1+\cdots+z^{N-1}\right) a^{[1]}(z)$. The scheme $\widetilde{S}$ with symbol $\widetilde{a}(z)$ has norm $\|\widetilde{S}\|=N\|S\|$. The inequality $\|S\| \leq\left\|S_{1}\right\|$ now follows from

$$
\|\widetilde{S}\|=\max _{k=1 \ldots N} \sum_{l \in \mathbb{Z}}\left|\widetilde{a}_{k-N l}\right| \leq \max _{k=1 \ldots N} \sum_{l \in \mathbb{Z}} \sum_{j=0}^{N-1}\left|a_{k-N l-j}^{[1]}\right|=\sum_{i=0}^{N-1} \sum_{l \in \mathbb{Z}}\left|a_{i-N l}^{[1]}\right| \leq N\left\|S_{1}\right\| .
$$

A crucial result in the convergence analysis of linear schemes is 
Lemma 2. For an affinely invariant linear scheme $S$ of finite mask there is a constant $C$ such that for all $p$ and $j>0$ we have

$$
\left\|\mathcal{F}_{j+1}(S p)-\mathcal{F}_{j}(p)\right\|_{\infty} \leq C d(p) .
$$

This follows e.g. from Equations (3.8)-(3.10) of [7]. For the convenience of the reader we give a proof here. It uses the following fact: Polynomial division of $\sum_{j=0}^{m} a_{j} z^{j}$ by $z^{N}-1$ yields the remainder $\sum_{j=0}^{N-1}\left(\sum_{i \in \mathbb{Z}} a_{j+i N}\right) z^{j}$, where we let $a_{j}=0$ in case $j<0$ or $j>m$.

Proof. Let $U$ denote the piecewise linear interpolatory subdivision rule with the same dilation factor $N$ as the given scheme $S$ and symbol $u(z)=(1+z+$ $\left.\cdots z^{N-1}\right)^{2} /\left(N z^{N-1}\right)=\sum_{i} u_{i} z^{i}$. By construction, $\mathcal{F}_{j+1}(U p)=\mathcal{F}_{j}(p)$ for all $j, p$. The mask of $S$ is denoted by $a(z)$. Because of $(2.4), \sum_{i}\left(a_{l-N i}-u_{l-N i}\right)=0$ for all $l$, which implies that $u(z)-a(z)$ is divisible by $1-z^{N}$. So we let $u(z)-a(z)=$ $e(z)\left(1-z^{N}\right) / z^{N}$, where $e(z)$ is the symbol of some subdivision scheme $E$. As $\Delta p(z)=(1-z) p(z) / z$, we have $(S-U) p=E \Delta p$ for all $p$. It follows that $\left\|\mathcal{F}_{j+1}(S p)-\mathcal{F}_{j}(p)\right\|=\left\|\mathcal{F}_{j+1}(S-U) p\right\|=\|(S-U) p\|_{\infty}=\|E \Delta p\|_{\infty} \leq\|E\| \cdot\|\Delta p\|_{\infty}$. We see that we can let $C=\|E\|$.

2.5. Proximity conditions. In this subsection we present the inequalities which quantify the differences between linear subdivision schemes " $S$ " of known properties and nonlinear schemes " $T$ " to be analyzed. The conditions consist of several inequalities: one measuring the distance of schemes $S, T$ (the actual proximity), and another one, which relates the coefficients $\mu_{i}$ of (2.10) to the exponents used in the proximity condition. We first give a general definition and immediately afterwards specialize it.

In order to explain why the proximity conditions of Def. 3 have the form presented here, a few comments are perhaps in order. In [22], $C^{1}$ smoothness of nonlinear subdivision schemes is shown by means of a proximity inequality of the form $\left\|S p_{i}-T p_{i}\right\| \leq C \cdot d(p)^{2}$, where $C$ e.g. depends on the surface we work in. For $C^{2}$ smoothness, inequalities which bound $\left\|\Delta T p_{i}-\Delta S p_{i}\right\|$ are needed. While it would have been nice to give an upper bound in terms of $d(\Delta p)^{2}$ or $d(p) d(\Delta p)$, it turned out that an upper bound uses a linear combination of $d(p)^{3}$ and $d(p) d(\Delta p)$. The fact that the upper bounds which are needed in order to establish higher order smoothness do not appear to have a simple form, led to consider the most general bounds available - any linear combination of products of the quantities $d(p), d(\Delta p)$, and so on.

While [22] considered only an upper bound of the form $\left\|S p_{i}-T p_{i}\right\| \leq d(p)^{\alpha_{0}}$ with $\alpha_{0}=2$, the present paper allows such exponents to vary freely. Indeed the $C^{1}$ theory remains true for any exponent $\alpha_{0}>1$, provided the decay rate $\mu_{0}$ is not too big (I am indebted to Adi Levin for this remark). As $\alpha$ approaches 1 , the maximum possible decay rate $\mu_{0}$ nears $1 / N$. It is only to be expected that for higher order smoothness there is an analogous relation between the exponents used in comparing $S$ and $T$ on the one hand, and the decay rates $\mu_{i}$ of $S$ on 
the other. The precise form of that relation (see (2.19) below) ensures that the proofs of Th. 5 and Th. 6 go through. Thus (2.19) might look artificial. There is however a reason that in some sense (2.19) is "right": This is the fact that the inequality in question appears to be just sufficient for both of the proofs.

Definition 3. Subdivision schemes $S, T$ satisfy a general proximity condition $(G P C)$ of order $k-1$, if for every $j$ with $0 \leq j<k$ there exist $C>0$ and a finite collection $\mathcal{A}_{j}$ of points $\boldsymbol{\alpha}=\left(\alpha_{0}, \ldots, \alpha_{j}\right) \in \mathbb{R}^{j+1}$ with $\alpha_{i} \geq 0$ and $\alpha_{0}+\cdots+\alpha_{j}>1$ such that for $j=0, \ldots, k$, and for all polygons in a certain class $\mathcal{P}_{M, \delta}$,

$$
\left\|\Delta^{j} S p-\Delta^{j} T p\right\|_{\infty} \leq C \sum_{\boldsymbol{\alpha} \in \mathcal{A}_{j}} d(p)^{\alpha_{0}} \cdots d\left(\Delta^{j} p\right)^{\alpha_{j}},
$$

and such that $S$ satisfies (2.9), (2.10) and there are strict upper bounds $\mu_{i}^{*}$ on the decay rates $\mu_{i}$ of (2.9) and (2.10) satisfying

$$
\left(\frac{\mu_{0}^{*}}{N^{0}}\right)^{\alpha_{0}} \cdots\left(\frac{\mu_{j-1}^{*}}{N^{j-1}}\right)^{\alpha_{j-1}} \leq \frac{\mu_{j}^{*}}{N^{j}}, \quad \boldsymbol{\alpha} \in \mathcal{A}_{j-1}, 1 \leq j \leq k
$$

Note that if $S, T$ satisfy a GPC of order $k$, they satisfy a GPC of any lower order. The special cases $\mathcal{A}_{0}=\{(2)\}$ and $\mathcal{A}_{1}=\{(3,0),(1,1)\}$, which correspond to curves on surfaces (see Section 4 ) are given below in Def. 4 .

The meaning of the inequality (2.19) is roughly as follows: According to Lemma 1 , the decay rates $\mu_{i}$ in the smoothness conditions are not smaller than $1 / N$, which is in some sense an optimal value (it is achieved by the Lane-Riesenfeld schemes). Other schemes may have values close to 1. Equation (2.19) says that the closer the coefficients $\mu_{i}$ are to 1 , the higher the exponents $\alpha_{i}$ must be.

Definition 4. Subdivision schemes $S, T$ satisfy a proximity condition of order 0 if there is $C>0$ such that for all polygons in a certain class $\mathcal{P}_{M, \delta}$,

$$
\begin{aligned}
& T p \in \mathcal{P}_{M, \delta},\|S p-T p\|_{\infty} \leq C d(p)^{2}, \quad \text { and } \\
& \mu_{0}^{* 2} \leq \frac{\mu_{1}^{*}}{N} .
\end{aligned}
$$

Subdivision schemes S, T satisfy a proximity condition of order 1 , if (2.20) and (2.21) hold, and if

$$
\begin{aligned}
& \|\Delta S p-\Delta T p\|_{\infty} \leq C\left[d(p) d(\Delta p)+d(p)^{3}\right], \quad \text { and } \\
& \mu_{0}^{* 3} \leq \frac{\mu_{2}^{*}}{N^{2}}, \quad \mu_{0}^{*} \mu_{1}^{*} \leq \frac{\mu_{2}^{*}}{N}
\end{aligned}
$$

In [22], the proximity condition (2.20) is established for certain analogues of linear schemes. The second part of the present paper is concerned with showing that (2.22) holds for analogues of a certain class of "factorizable" linear schemes. Depending on its decay rates $\mu_{i}$, such a linear scheme may or may not fulfill (2.23). In case it does not, there is still the possibility that by replacing the linear scheme $S$ by an iterated scheme $S^{m}$, the general proximity condition is satisfied. The phenomenon that for purposes of smoothness analysis one has 
to consider iterated schemes $S^{m}$ rather than $S$ itself is common also for linear schemes.

We therefore ask: If $S, T$ fulfill a proximity condition, is the same true for the iterates $S^{m}, T^{m}$ ? In order not to overburden the reader, we give an answer only for a special case:

Lemma 3. If $S, T$ fulfill (2.20) for all $p$ with $d(p) \leq \delta$, then the same is true for the iterates $S^{m}, T^{m}$, provided the first derived scheme $S_{1}$ exists (which is true for all affinely invariant schemes). An analogous result is true for (2.22), provided the derived scheme $S_{2}$ exists.

Proof. The existence of $S_{1}$ and $S_{2}$ means that there are $\mu_{0}, \mu_{1}$ with $d(S p) \leq \mu_{0} d(p)$ and $d(\Delta S p) \leq \mu_{1} d(\Delta p)$. The result is shown by induction. Assume that it is true for $S^{m-1}$ and $T^{m-1}$. The letter $C$ is used indiscriminantly for any constant. We use $d(p) \leq \delta$ to deduce $d(p)^{k} \leq d(p) \delta^{k-1}$ and the inequality $d(q) \leq d(p)+2\|p-q\|$ to compute $\left\|S^{m} p-T^{m} p\right\| \leq\left\|S^{m} p-S T^{m-1} p\right\|+\left\|S T^{m-1} p-T^{m} p\right\| \leq\|S\| \| S^{m-1} p-$ $T^{m-1} p \|+C d\left(T^{m-1} p\right)^{2} \leq C d(p)^{2}+C\left(d\left(S^{m-1} p\right)+2\left\|T^{m-1} p-S^{m-1} p\right\|\right)^{2} \leq C d(p)^{2}+$ $C\left(\mu_{0}^{m} d(p)+2 C d(p)^{2}\right)^{2} \leq d(p)^{2}\left(C+C \delta+C \delta^{2}\right)$. The computation for (2.22) is similar but slightly more complicated.

The condition that $\alpha_{0}+\cdots+\alpha_{j}>1$ used in Def. 3 is sometimes fulfilled automatically:

Lemma 4. If $1 / N<\mu_{i}^{*}, \ldots, \mu_{j}^{*} \leq 1$ and (2.19) holds, then $\alpha_{0}+\cdots+\alpha_{j}>1$.

Proof. We let $\mu_{i}^{*}=N^{-m_{i}}$ and note that (2.19) now reads

$$
\alpha_{0}\left(m_{0}+0\right)+\cdots+\alpha_{j-1}\left(m_{j-1}+(j-1)\right) \geq m_{j}+j .
$$

The assumption $1 / N<\mu_{i}^{*} \leq 1$ implies that $0 \leq m_{i}<1$. We look for points $\boldsymbol{\alpha}^{(i)}=\left(0, \ldots, 0, \alpha_{i}, 0, \ldots, 0\right)$ with $0 \leq i<j$ on the coordinate axes of $\mathbb{R}^{j}$ for which (2.24) is an equality and show that in that special case $\alpha_{i}>1$ :

$$
\alpha_{i}\left(m_{i}+i\right)=m_{j}+j \Longrightarrow \alpha_{i}=\frac{m_{j}+j}{m_{i}+i}>1 .
$$

We see that the distance of the points $\boldsymbol{\alpha}^{(0)}, \ldots, \boldsymbol{\alpha}^{(j-1)}$ from the origin is $>1$. It follows that within the sector $\alpha_{0}, \ldots, \alpha_{j} \geq 0$, the halfspace $\sum_{0 \leq i<j} \alpha_{i} \geq 1$ contains the halfspace given by (2.24) in its interior. Consequently, $\sum_{0 \leq i \leq j} \alpha_{j} \geq$ $\sum_{0 \leq i<j} \alpha_{j}>1$.

2.6. Convergence from proximity. In this section we review and partly extend results of [22] which concern convergence of perturbed schemes. The proofs are omitted, since the extension consists of replacing the exponent 2 by the exponent $\alpha$, and the proofs are completely analogous to the proofs given in [22].

Theorem 2. Suppose that S,T satisfy

$$
\|S p-T p\| \leq d(p)^{\alpha}, \quad \alpha>1
$$


and $S$ satisfies a convergence condition with factor $\mu_{0}<1$ for all $p \in \mathcal{P}_{M, \varepsilon}$. Then there is $\delta>0$ and $\bar{\mu}_{0}<1$ such that $T$ satisfies a convergence condition with factor $\bar{\mu}_{0}$ for all $p \in \mathcal{P}_{M, \delta}$. By choosing $\delta$ small enough, we can achieve that $\left|\bar{\mu}_{0}-\mu_{0}\right|$ is arbitrarily small.

Theorem 3. Assume that $S$ and $T$ meet the conditions of Th. 2. In the notation of Th. 2, for all $p \in \mathcal{P}_{M, \delta}$ and $f=\mathcal{F}_{0}(p)$, the sequence $T^{j} f$ converges to a continuous limit in the sup norm.

The following result compares the limits of a linear scheme and its nonlinear analogue. The uniform boundedness of $\left\|S^{i}\right\|$ mentioned in the statement of the theorem is guaranteed if $S$ is a convergent scheme.

Theorem 4. We assume that $S$ and $T$ fulfill the conditions of Th. 2, and that $\left\|S^{i}\right\|$ is uniformly bounded by $A>0$. Then for $p \in \mathcal{P}_{M, \delta}$,

$$
\left\|S^{\infty} p-T^{\infty} p\right\|_{\infty} \leq \frac{A C}{1-\bar{\mu}_{0}^{\alpha}} d(p)^{\alpha} .
$$

2.7. Deriving smoothness conditions for nonlinear schemes. In the following we establish that mixed smoothness conditions according to Def. 2 follow from the GPC of Def. 3.

Theorem 5. Suppose that $S$ satisfies the $k$-th order smoothness condition of Def. 2 and that $S, T$ satisfy an order $k-1$ GPC according to Def. 3, for all $p \in \mathcal{P}_{M, \varepsilon}$. Further assume that in the notation of Def. 3, $\mu_{0}^{*} \leq 1$. Then there is $\delta>0$ such that $T$ satisfies a mixed $k$-th order smoothness condition according to Def. 2 with factors $\bar{\mu}_{i}<\mu_{i}^{*}$, for all $p$ in $\mathcal{P}_{M, \delta}$.

Proof. We use the notation of Def. 2 and Def. 3: The smoothness condition uses the factors $\mu_{0}, \ldots, \mu_{k}$ with $\mu_{i}<\mu_{i}^{*}$, and the proximity is encoded in exponent collections $\mathcal{A}_{0}, \ldots, \mathcal{A}_{k-1}$. We want to show that there are factors $\bar{\mu}_{j}<\mu_{j}^{*}$ and nonnegative polynomials $\bar{P}_{j}$ such that

$$
d\left(N^{j l} \Delta^{j} T^{l} p\right) \leq \bar{\mu}_{j}^{l} \bar{P}_{j}(l) d(p), \quad \bar{P}_{j}(i) \geq 0 \text { if } i \geq 0
$$

holds for $j=0, \ldots, k$, any $l>0$, and all $p$ with $d(p)<\delta$. By Th. 2 and the assumption $\mu_{0}<1$, this is true in the case $k=0$. We proceed by induction, i.e., we assume that $(2.28)$ holds for $j=0, \ldots, k-1$. We let

$$
d_{l}=d\left(N^{k l} \Delta^{k} T^{l} p\right), \quad q=T^{l-1} p .
$$

The proximity condition (2.18) of order $k-1$ shows that for any $l \geq 1$,

$$
\begin{aligned}
d_{l} & =d\left(N^{k l} \Delta^{k} T q\right) \leq d\left(N^{k l} \Delta^{k} S q\right)+2 \cdot N^{k l}\left\|\Delta^{k} S q-\Delta^{k} T q\right\| \\
& \leq d\left(N^{k l} \Delta^{k} S q\right)+2 \cdot 2 \cdot N^{k l}\left\|\Delta^{k-1} S q-\Delta^{k-1} T q\right\| \\
& \leq \mu_{k} d\left(N^{k(l-1)} \Delta^{k} q\right)+4 N^{k l} C \sum_{\boldsymbol{\alpha} \in \mathcal{A}_{k-1}} \prod_{j} d\left(\Delta^{j} q\right)^{\alpha_{j}} \\
& =: \mu_{k} d_{l-1}+h .
\end{aligned}
$$


We have used that for all $p, q$ the relations $d(p) \leq d(q)+2\|p-q\|_{\infty}$ and $\|\Delta p\| \leq$ $2\|p\|$ hold.

The induction assumption (2.28) on the quantities $d\left(\Delta^{j} T^{l-1} p\right)$ for $j=0, \ldots, k-$ 1 is now used to replace them by their upper bounds. In this process, $N^{k l}$, as it occurs in $h$, is spread over several factors. We use the symbols $|\boldsymbol{\alpha}|=\sum_{j=0}^{k-1} \alpha_{j}$ and $w(\boldsymbol{\alpha})=\sum_{j=0}^{k-1} j \alpha_{j}$ for $\boldsymbol{\alpha} \in \mathcal{A}_{k-1}$.

$$
\begin{aligned}
& h=4 C \sum_{\boldsymbol{\alpha} \in \mathcal{A}_{k-1}} N^{k l-(l-1) w(\boldsymbol{\alpha})} \prod_{j=0}^{k-1} d\left(N^{j(l-1)} \Delta^{j} T^{l-1} p\right)^{\alpha_{j}} \\
& \leq 4 N^{k} C \sum_{\boldsymbol{\alpha} \in \mathcal{A}_{k-1}} N^{(l-1)(k-w(\boldsymbol{\alpha}))} \prod_{j=0}^{k-1}\left(\bar{\mu}_{j}^{l-1} d(p) \bar{P}_{j}(l-1)\right)^{\alpha_{j}} \\
& \leq 4 N^{k} C \sum_{\boldsymbol{\alpha} \in \mathcal{A}_{k-1}}\left(N^{k} \prod_{j=0}^{k-1}\left(\bar{\mu}_{j} / N^{j}\right)^{\alpha_{j}}\right)^{l-1} d(p)^{|\boldsymbol{\alpha}|} \widetilde{P}(l-1) .
\end{aligned}
$$

Here $\widetilde{P}$ is the polynomial $\widetilde{P}=\prod_{j=0}^{k-1} \bar{P}_{j}^{\left\lceil\alpha_{j}\right\rceil} . \quad \widetilde{P}$ is nonnegative. Let $\widetilde{\mu}_{k}:=$ $N^{k} \max _{\boldsymbol{\alpha} \in \mathcal{A}_{k-1}} \prod\left(\bar{\mu}_{j} / N^{j}\right)^{\alpha_{j}}$. By the induction assumption $\bar{\mu}_{j}<\bar{\mu}_{j}^{*}(j=0, \ldots, k-$ 1), (2.19) implies that $\widetilde{\mu}_{k}<\mu_{k}^{*}$. Recall that $d(p)<\delta$ :

$$
\begin{aligned}
& h \leq 4 N^{k} C \sum_{\boldsymbol{\alpha} \in \mathcal{A}_{k-1}} \widetilde{\mu}_{k}^{l-1} d(p)^{|\boldsymbol{\alpha}|} \widetilde{P}(l-1) \\
& \leq d(p) \widetilde{\mu}_{k}^{l-1} \cdot 4 N^{k} C \sum_{\boldsymbol{\alpha} \in \mathcal{A}_{k-1}} \delta^{|\boldsymbol{\alpha}|-1} \widetilde{P}(l-1)=\widetilde{\mu}_{k}^{l-1} d(p) P_{k-1}^{*}(l),
\end{aligned}
$$

with a nonnegative polynomial $P_{k-1}^{*}$. We are going to show by induction that

$$
d_{l} \leq \mu_{k}^{l} d_{0}+d(p) \sum_{j=1}^{l} \mu_{k}^{l-j} P_{k-1}^{*}(j) \widetilde{\mu}_{k}^{j-1} .
$$

Indeed (2.33) is true for $l=0$. If (2.33) is true for $l-1$, then

$$
\begin{aligned}
& d_{l} \leq \mu_{k} d_{l-1}+h \leq \mu_{k}\left[\mu_{k}^{l-1} d_{0}+d(p) \sum_{j=1}^{l-1} \mu_{k}^{l-1-j} P_{k-1}^{*}(j) \tilde{\mu}_{k}^{j-1}\right] \\
& +\widetilde{\mu}_{k}^{l-1} d(p) P_{k-1}^{*}(l)=\mu_{k}^{l} d_{0}+d(p) \sum_{j=1}^{l} \mu_{k}^{l-j} P_{k-1}^{*}(j) \widetilde{\mu}_{k}^{j-1} .
\end{aligned}
$$

This concludes the proof of (2.33). We define $\bar{\mu}_{k}:=\max \left\{\mu_{k}, \widetilde{\mu}_{k}\right\}<\mu_{k}^{*}$ and consider the polynomial $P_{k}$ defined by $\sum_{i=1}^{j} P_{k-1}^{*}(i)=P_{k}(j)$, which is nonegative. With these definitions, (2.33) implies that

$$
d_{l} \leq \bar{\mu}_{k}^{l} d_{0}+\bar{\mu}_{k}^{l-1} d(p) \sum_{j=1}^{l} P_{k-1}^{*}(j)=\bar{\mu}_{k}^{l} d_{0}+d(p) \bar{\mu}_{k}^{l-1} P_{k}(l) .
$$

Since $d\left(\Delta^{k} p\right) \leq 2^{k} d(p),(2.34)$ implies the inequality

$$
d_{l} \leq \bar{\mu}_{k}^{l}\left[d\left(\Delta^{k} p\right)+d(p) P_{k}(l) / \bar{\mu}_{k}\right] \leq \bar{\mu}_{k}^{l}\left(2^{k}+P_{k}(l) / \bar{\mu}_{k}\right) d(p),
$$

for $d(p)<\delta$. We let $\bar{P}_{k}(l)=2^{k}+\bar{\mu}_{k}^{-1} P_{k}(l)$, and the proof is complete.

It is obvious that for any given $\mu_{0}, \ldots, \mu_{k}$ with $\mu_{0}<1$ and exponent collections $\mathcal{A}_{0}, \ldots, \mathcal{A}_{k-1}$ we can choose $\mu_{0}^{*}, \mu_{1}^{*}, \ldots$ such that general proximity holds true, if we do not have to obey any further inequalities concerning the magnitude of $\mu_{i}^{*}$. It follows that with Th. 5 we can derive a mixed smoothness condition for any nonlinear scheme $T$ which satisfies (2.18). 
2.8. Smoothness of limit curves. It is well known that $C^{k}$ smoothness of the limit curve $T^{\infty} f$ as defined by (2.7) follows from existence of the limits

$$
\lim _{l \rightarrow \infty} \mathcal{F}_{l}\left(N^{j l} \Delta^{j} T^{l} p\right), \quad j=0, \ldots, k,
$$

with respect to the sup norm, provided those limits are continuous Such a limit then equals the $j$-th derivative of the curve $T^{\infty} f$. It is obviously sufficient to prove the result in the case $k=1$ (see e.g. [3]). We state this result in a form which directly applies to our setting.

Lemma 5. Assume that the sequence $p^{l}$ of polygons has the property that $\lim _{l \rightarrow \infty}$ $\mathcal{F}_{l}\left(p^{l}\right)=f$ with respect to the sup norm. We let

$$
g_{j, l}:=\mathcal{F}_{l}\left(N^{j l} \Delta^{j} p^{l}\right)
$$

If $g_{j, l}$ is a Cauchy sequence, and $\lim _{l \rightarrow \infty} d\left(N^{j l} \Delta^{j} p^{l}\right)=0$ for $j=1, \ldots, k$, then $f$ is $C^{k}$ with $f^{(j)}=\lim _{l \rightarrow \infty} g_{j, l}$ (with respect to the sup norm).

Theorem 6. If under the conditions of Th. 5, $\mu_{0}^{*}, \ldots, \mu_{k}^{*} \leq 1$ and the symbol of $S$ is divisible by $\left(1+z+\cdots+z^{N-1}\right)^{k+1}$, then the limit curves $T^{\infty} p$ are $C^{k}$ for all polygons $p$ such that $T^{l} p$ converges.

Proof. The case $k=0$ is Th. 3. The case $k=1$ has been shown in [22]. Here we show the general case $k>0$ by using the proximity condition of order $k-1$ :

$$
\left\|\Delta^{k-1} S p-\Delta^{k-1} T p\right\| \leq C \sum_{\boldsymbol{\alpha} \in \mathcal{A}_{k-1}} \prod d\left(\Delta^{j} p\right)^{\alpha_{j}} .
$$

By Th. 5, a mixed smoothness condition holds for $T$ : there are nonnegative polynomials $P_{j}$ and factors $\mu_{j}$ for $j=1, \ldots, k$ such that

$$
\left\|\Delta^{j} T^{l} p\right\| \leq N^{-j l} P_{j}(l) \bar{\mu}_{j}^{l} d(p),
$$

such that $\bar{\mu}_{i}<\mu_{i}^{*}(i=0, \ldots, k)$. For a linear subdivision scheme $S$ and its $k$ th derived scheme $S_{k}$, a smoothness condition of order $k$ in the sense of Def. 2 which holds for $S$ is nothing but a continuity condition for $S_{k}$. According to our assumptions, the symbol of $S_{k}$ is divisible by $1+z+\cdots+z^{N-1}$, so $S_{k}$ is affinely invariant. By Lemma 2, there is a constant $\widetilde{C}$ such that

$$
\left\|\mathcal{F}_{j+1}\left(S_{k} \Delta^{k} p\right)-\mathcal{F}_{j}\left(\Delta^{k} p\right)\right\|_{\infty} \leq \widetilde{C} d\left(\Delta^{k} p\right) .
$$

We consider the sequence $\mathcal{F}_{l}\left(N^{k l} \Delta^{j} T^{l} p\right)$. With $q:=T^{l} p$,

$$
\begin{aligned}
& \left\|\mathcal{F}_{l+1} N^{k(l+1)} \Delta^{k} T^{l+1} p-\mathcal{F}_{l} N^{k l} \Delta^{k} T^{l} p\right\|_{\infty} \\
\leq & \left\|\mathcal{F}_{l+1} N^{k(l+1)} \Delta^{k}(T-S) q\right\|+\left\|\left(\mathcal{F}_{l+1} N^{k} \Delta^{k} S-\mathcal{F}_{l} \Delta^{k}\right) N^{k l} q\right\| \\
\leq & 2 N^{k(l+1)}\left\|\Delta^{k-1}(T-S) q\right\|+\left\|\left(\mathcal{F}_{l+1} S_{k}-\mathcal{F}_{l}\right) \Delta^{k} N^{k l} q\right\|=(*)+(* *)
\end{aligned}
$$

We use (2.38) and (2.39) and compute for any $\boldsymbol{\alpha} \in \mathcal{A}_{k-1}$ :

$$
\begin{aligned}
& N^{k(l+1)} \prod_{j} d\left(\Delta^{j} q\right)^{\alpha_{j}}=N^{k(l+1)} \prod_{j}\left[N^{-j l} d\left(N^{j l} \Delta^{j} T^{l} p\right)\right]^{\alpha_{j}} \\
& \leq N^{k} d(p)^{|\boldsymbol{\alpha}|}\left(N^{k-w(\boldsymbol{\alpha})} \prod_{j} \bar{\mu}_{j}^{\alpha_{j}}\right)^{l} \prod_{j}\left(P_{j}(l)\right)^{\alpha_{j}}
\end{aligned}
$$



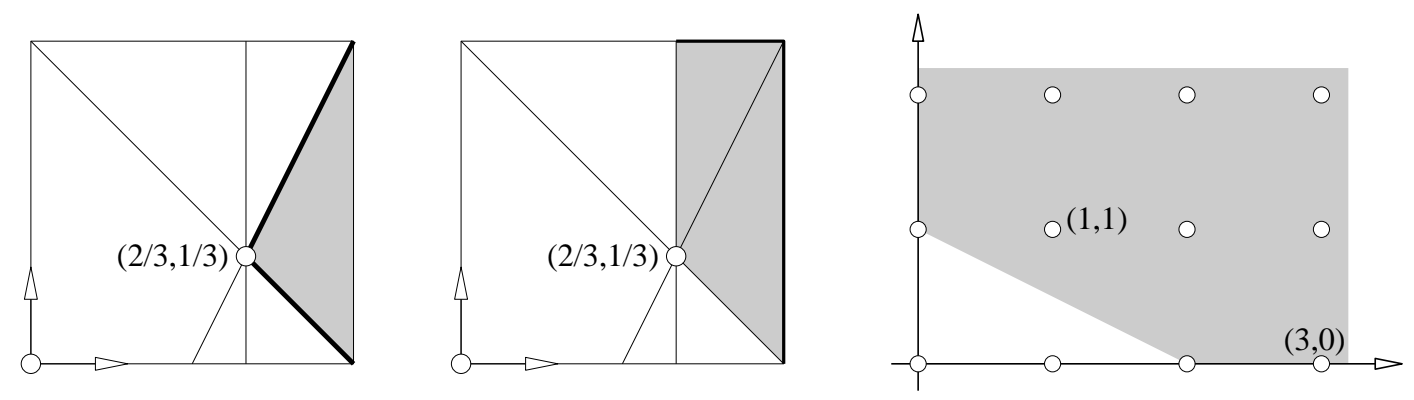

Figure 1. Admissible factors $\left(\mu_{0}^{*}, \mu_{1}^{*}\right)$ (left) and $\left(\mu_{0}, \mu_{1}\right)$ (center) in the $\left(m_{0}, m_{1}\right)$-plane (see text) for the proximity conditions of Def. 4. Right: Admissible exponents $\alpha_{0}, \alpha_{1}$ for $\mu_{0}^{*}=\mu_{1}^{*}=1 / N, \mu_{2}<1$.

(2.38) and (2.19) show that (2.41) implies

$$
(*) \leq 2 C N^{k} \sum_{\boldsymbol{\alpha} \in \mathcal{A}_{k-1}} d(p)^{|\boldsymbol{\alpha}|} \bar{\mu}_{k}^{l} \prod_{j}\left(P_{j}(l)\right)^{\alpha_{j}} .
$$

In order to estimate $(* *)$, we use (2.40) and (2.39)

$$
(* *) \leq \widetilde{C} N^{k l} d\left(\Delta^{k} q\right) \leq \widetilde{C} \bar{\mu}_{k}^{l} P_{k}(l) d(p) .
$$

As $\mu_{k}^{*} \leq 1$, we have shown that $\mathcal{F}_{l}\left(N^{j l} \Delta^{j} T^{l} p\right)(l \rightarrow \infty)$ is a Cauchy sequence for $j=1, \ldots, k$. Furthermore, the smoothness condition which holds for $T$ says that $\lim _{l \rightarrow \infty} d\left(N^{j l} \Delta^{j} T^{l} p\right)=0$ for $j=0, \ldots, k$. Thus Lemma 5 applies, and the proof is complete.

\section{EXAMPLES}

The next sections treat subdivision in manifolds, which is an important context where nonlinear analogues of linear schemes occur. Before that, we dicuss some facts which easily follow from the results obtained so far.

3.1. Proximity of orders $\mathbf{0}$ and $\mathbf{1}$. If a linear scheme $S$ is given, (2.19) in the general proximity condition and the inequalities $\mu_{0}^{*}, \ldots, \mu_{k}^{*} \leq 1$ required by Th. 6 put restrictions on the exponents $\alpha_{i}$ used in the GPC. On the other hand, if a certain way of perturbing linear schemes is known to yield inequalities like (2.18) or (2.20) or (2.22), such that the exponents $\alpha_{i}$ are known, we might ask which linear schemes have factors $\mu_{i}$ meeting the requirements of (2.19). By letting $\mu_{i}=N^{-m_{i}},(2.19)$ turns into the linear inequality (2.24). The inequalities $\mu_{i} \geq 1 / N$ from Lemma 1 and the requirements $\mu_{i}<1$ yield $0<m_{i} \leq 1$.

We first have a look at the zero order proximity condition of Def. 4. The requirement $\mu_{1}<1$ is fulfilled by letting $\mu_{1}^{*}=1$, and we arrive at the two equivalent conditions

$$
\mu_{0}^{\alpha_{0}}<1 / N \Longleftrightarrow \alpha_{0}>1 / m_{0} .
$$


We see that for any smooth scheme $S$ there is a general proximity condition of the form $\|S p-T p\|_{\infty} \leq d(p)^{\alpha_{0}}$ which ensures smoothness of the scheme $T$. Conversely, if such a condition is given, it applies to $S$ only if $\mu_{0} \leq N^{-1 / \alpha_{0}}$.

As to first order proximity conditions, we force $\mu_{2}<1$ by letting $\mu_{2}^{*}=1$. Then (2.19) yields

$$
\alpha_{0} m_{0}-\alpha_{1}\left(m_{1}+1\right)>2, \quad \text { for all }\left(\alpha_{0}, \alpha_{1}\right) \in \mathcal{A}_{1} .
$$

In the concrete case of the zero order and first order proximity stated in Def. 4 , the inequalities (3.1) and (3.2) for $\alpha_{0}=2$ and $\alpha_{1} \in\{(1,1),(3,0)\}$ define a certain domain for admissible factors $\mu_{0}^{*}, \mu_{1}^{*}$ and $\mu_{0}, \mu_{1}$ which are depicted in Figure 1, left and center.

The other way round, for a given scheme with, e.g., $\mu_{0}=\mu_{1}=1 / N$, the admissible exponents $\alpha_{0}, \alpha_{1}$ used in the GPC are shown by Fig. 1, right.

3.2. Sharpness of the proximity conditions. The general proximity condition, especially (2.19) is sufficient for $C^{k}$ smoothness of a scheme $T$, provided the scheme $S$ fulfills the requirements of Th. 6 , but it is possible that (2.19) can be improved so as to be less restrictive. In the following we show that for the case of B-spline schemes and a special type of proximity, the inequalities (2.19) are necessary for $C^{k}$ smoothness: It is easy to see that in the case $\mu_{0}=\cdots=\mu_{k}=1 / N$ and

$$
\left\|\Delta^{j} S p-\Delta^{j} T p\right\| \leq C d\left(\Delta^{j} p\right)^{\alpha}, \quad j=1, \ldots, k-1,
$$

any $\alpha>1$ implies $C^{k}$ smoothness of $T$, but $\alpha=1$ does not. A counterexample is given by the B-spline schemes of (2.2): Let $S=S_{(k+1)}, T=S_{(k)}$ and consider the symbols $a(z)=(1+z)^{k+2} /(2 z)^{k+1}$ and $b(z)=(1+z)^{k+1} /(2 z)^{k}$ of $S$ and $T$, respectively, as in (2.16). With $U=S-T$ the $j$-th derived scheme $U_{j}$ has the symbol $u^{[j]}(z)=e^{(j)}(z)\left(1-z^{2}\right) / z^{2}$ with $e^{(j)}(z)=z((1+z) / 2 z)^{k-j}$. This implies that $U_{j} p=E^{(j)} \Delta p$, where $E^{(j)}$ has the symbol $e^{(j)}(z)$. We compute

$$
\Delta^{j} S p-\Delta^{j} T p=\Delta^{j} U p=\frac{1}{2^{j}} U_{j} \Delta^{j} p=\frac{1}{2^{j}} E^{(j)} \Delta^{j+1} p
$$

It follows that

$$
\left\|\Delta^{j} S p-\Delta^{j} T p\right\| \leq \frac{1}{2^{j}}\left\|E^{(j)}\right\| d\left(\Delta^{j} p\right), \quad j=0, \ldots, k .
$$

As $S$ is $C^{k}$, but $T$ is not, (3.5) cannot be a valid proximity condition implying $C^{k}$ smoothness.

3.3. Examples of perturbed schemes of $C^{k}$ smoothness. Suppose that $S$ is a linear scheme with $C^{k}$ limits and whose $(k+1)$-st derived scheme exists, i.e., whose symbol is divisible by $\left(1+z+\cdots+z^{N-1}\right)^{k+1}$, as required by Th. 6 . A perturbed scheme $T$ of the form

$$
T p_{i}=S p_{i}+Q(p, i) d(p)^{\alpha}, \quad\|Q(p, i)\| \leq C,
$$


with $\alpha>1$ and arbitrary bounded $Q$ satisfies the inequality

$$
\left\|\Delta^{j} T p-\Delta^{j} S p\right\| \leq 2^{j}\|S p-T p\| \leq 2^{j} C d(p)^{\alpha} .
$$

We consider the factors $\mu_{i}=\frac{1}{N}\left\|S_{i+1}\right\|$ which appear in the smoothness conditions for $S$, let $\mu_{i}=N^{-m_{i}}$ and look at (2.19) for the special case that the exponent lists have the form $\mathcal{A}_{0}=\{(\alpha)\}, \mathcal{A}_{1}=\{(\alpha, 0)\}, \ldots, \mathcal{A}_{k-1}=\{(\alpha, 0, \ldots, 0)\}$ (i.e., we reformulate (3.7) to fit the formalism of Def. 3). $S$ and $T$ fulfill a general proximity condition of order $k-1$, if, according to $(2.19), \mu_{0}^{* \alpha_{0}} \leq \mu_{j}^{*} N^{-j}$ for $0 \leq j<k$, i.e., if

$$
\alpha_{0} m_{0}>m_{j}+j, \quad j=0, \ldots, k-1, \quad \alpha_{0} m_{0}>k .
$$

This is equivalent to $\alpha>\left(j+m_{j}\right) / m_{0}$ for $j=0, \ldots, k-1$ and $\alpha>k / m_{0}$. With $1 / N \leq \mu_{j}<1$ this is further equivalent to

$$
\alpha>k / m_{0}=k \log N /\left|\log \mu_{0}\right| .
$$

We see that if $\alpha$ fulfills the inequality (3.9), $T$ has $C^{k}$ limit curves.

\section{Curve schemes And Their analogous SChemes in manifolds}

This section continues the discussion of proximity conditions in [22], which was solely concerned with zero order proximity. The representation of affinely invariant linear subdivision schemes in terms of averages, which exists in general by Th. 1 and is for the B-spline schemes demonstrated by (2.2), is used to define nonlinear schemes on manifolds in two different ways.

One way is to replace affine averages by geodesic averages. The second consists of projecting affine averages onto the manifold. These constructions of nonlinear schemes from linear ones apply to surfaces, to certain Lie groups, in particular to the Euclidean motion group, and to abstract Riemannian manifolds.

A general analysis of certain subdivision schemes on abstract Riemannian manifolds is done in [17], [16], and [18], where the geodesic analogues of the second and third degree B-spline schemes are shown to converge to smooth curves with Lipschitz derivatives, and that the limit curves of the second degree algorithm are not even piecewise $C^{2}$ in general.

4.1. Geodesic averages in surfaces and geodesic subdivision. Here we review briefly the construction of analogous geodesic and group schemes in surfaces and matrix groups, which is presented in more detail in [22].

We replace the straight lines of affine space by the geodesic lines in a surface or Riemannian manifold and the average of two points by a corresponding point on the geodesic. We study this concept first for surfaces only. The reason for this is that our method of analyzing smoothness of nonlinear schemes requires comparison with linear schemes, and for our proofs the ambient space where a surface is immersed in is necessary. We consider abstract Riemannian manifolds only in the very end. 
The surfaces we deal with and the Euclidean spaces they are contained in have arbitrary dimensions, especially because of the proof of Cor. 1 later. Curvature theory of such surfaces employs the vector-valued second fundamental form " $\Pi(v, w)$ " of two tangent vectors $v, w$ attached to a point $p \in M$ (cf. [6], § 6.2). Its definition is as follows: Suppose that $c(t)$ is a curve in $M$ with $c\left(t_{0}\right)=0$ and $\dot{c}\left(t_{0}\right)=v$, and further suppose that $w(t)$ is a tangent vector field along the curve $c(t)$ such that $w\left(t_{0}\right)=w$. Then

$$
\Pi_{p}(v, w)=\left.(d w / d t)^{\perp}\right|_{t=t_{0}},
$$

where the symbol " $\perp$ " means the component of a vector orthogonal to the surface's tangent plane $T_{p} M$. It can be shown that $\Pi_{p}$ is well defined, bilinear, and symmetric. The well known defining property " $\ddot{c}$ orthogonal to the surface" of a geodesic line $c(t)$ obviously can be reworded as

$$
\ddot{c}\left(t_{0}\right)=\Pi_{c\left(t_{0}\right)}\left(\dot{c}\left(t_{0}\right), \dot{c}\left(t_{0}\right)\right)
$$

(let $v=\dot{c}\left(t_{0}\right)$ and $\left.w(t)=\dot{c}(t)\right)$. Following $\oint 2$ of [22], we define

Definition 5. If $c$ is the unique shortest geodesic which joins $x$ and $y$, then we let

$$
g-a v_{\alpha}(x, y):=c(\alpha t), \text { if } c(0)=x, c(t)=y .
$$

The geodesic analogue $T$ of an affinely invariant linear scheme $S$, which is expressed in terms of averages, is defined by replacing each occurrence of the av operator by the g-av operator.

Note that both affine and geodesic averages fulfill the relations

$$
\operatorname{av}_{1-\alpha}(y, x)=\operatorname{av}_{\alpha}(x, y), \quad g-\operatorname{av}_{1-\alpha}(y, x)=g-\operatorname{av}_{\alpha}(x, y) .
$$

4.2. Averages in matrix groups. This subsection extends the concept of geodesic subdivision to matrix groups, such that, e.g. for the group of Euclidean motions, the helical motions appear as geodesic-like curves (cf. [2] or [13]). This means e.g. that the geodesic midpoint of two positions of a rigid body is found by first determining the shortest helical motion which transforms the first position (at time $t=0$ ) into the other (at time $t=\tau$ ), and then evaluating this helical motion half way in between, i.e., at $t=\tau / 2$.

In general, we use the left translates of one-parameter subgroups of matrix groups and general Lie groups as geodesics. In terms of the matrix exponential function, these curves can be written as

$$
c(t)=g \exp (t v)
$$

where $g v$ is tangent to the group in the point $c(0)=g$. This is discussed in more detail in sections 2.3 and 6.3 of [22]. We are able to treat matrix groups in the same way as surfaces, if we can show that the geodesics in matrix groups fulfill a differential equation similar to the surface case. 
Remark: The usage of the word 'geodesic' in the Lie group context does not mean that the Lie group carries a metric such that the geodesics defined by (4.5) are geodesics in the Riemannian sense.

Definition 6. A Lie group of $n \times n$ matrices is called of constant velocity, if there is a Euclidean metric in the $n^{2}$-dimensional space of matrices, such that the geodesics defined by (4.5) are traversed with constant velocity.

It is shown in [22] that all subgroups of the orthogonal group, indeed all compact groups, and also the Euclidean motion group, are of constant velocity. As to a differential equation of geodesics, that paper contains the following

Lemma 6. Assume that $G$ is a Lie group of $n \times n$ matrices. Then the geodesics are precisely the solution curves of the differential equation

$$
\ddot{c}=B_{c(t)}(\dot{c}(t), \dot{c}(t)), \text { with } B_{g}(v, w)=\frac{1}{2}\left(v g^{-1} w+w g^{-1} v\right) .
$$

4.3. Projection subdivision. The method of projection is a very general way of introducing nonlinearity.

Definition 7. Consider a submanifold $M$ of $\mathbb{R}^{n}$. A generalized projection $P$ onto $M$ is a smooth mapping onto $M$ defined in a neighbourhood of $M$, such that $P(x)=x$ for all $x \in M$.

How smooth exactly $P$ must be depends on the application. One example of a projection is the orthogonal projection onto $M$.

Definition 8. The projection analogue $T$ of an affinely invariant linear scheme $S$, which is expressed in terms of averages, is defined by replacing each occurrence of the av operator by "Pav".

Examples of projections which are readily computable are the gradient flow towards general level set surfaces [23], and orthogonal projection onto selected surfaces like spheres, tori, or the Euclidean motion group (see [1, 23]). A further application of the projection method is the perturbation of subdivision schemes by obstacles [23].

4.4. Taylor's formula. Verification of proximity conditions for subdivision schemes on manifolds is based on Taylor's formula. For the convenience of the reader, we repeat it here, using the notation we employ later: If $P: U \subset \mathbb{R}^{n} \rightarrow \mathbb{R}^{m}$ is a mapping of sufficient smoothness, then for all $x$ and $h$ such that the line segment connecting $x$ and $h$ lies in the domain of $P$, we have

$$
P(x+h)=P(x)+\sum_{j=1}^{k} \frac{1}{j !} d_{x}^{j} P(\underbrace{h, \ldots, h}_{j})+\frac{d_{x+\theta h}^{k+1} P(h, \ldots, h)}{(k+1) !},
$$


with $0<\theta<1$. The $k$-th derivative $d_{x}^{k} P$ of $P$ in the point $x$ is a $k$-linear mapping. If the vector $u_{i}$ has coordinates $\left(u_{i 1}, \ldots, u_{i n}\right)$, then

$$
d_{x}^{k} P\left(u_{1}, \ldots, u_{k}\right)=\sum_{i_{1}, \ldots, i_{k}=1}^{n} u_{1 i_{1}} \cdots u_{k i_{k}} \frac{\partial^{k} P(x)}{\partial x_{i_{1}} \cdots \partial x_{i_{k}}} .
$$

The operator norm of the $k$-th derivative is defined as

$$
\left\|d^{k} P\right\|:=\max \left\{\left\|d^{k} P\left(u_{1}, \ldots, u_{k}\right)\right\| \mid\left\|u_{i}\right\| \leq 1\right\} .
$$

We give some lemmas, which are needed later. The first one is quoted from [22].

Lemma 7. Assume that $c$ is a curve with $\|\dot{c}\|=1$ and $\|\ddot{c}\| \leq C$. Then

$$
\begin{aligned}
& \|c(0)+t \dot{c}(0)-c(t)\| \leq \frac{C t^{2}}{2}, \quad|t|-C t^{2} / 2 \leq\|c(t)-c(0)\|, \\
& t<1 / C \Longrightarrow|t| \leq 2\|c(t)-c(0)\| .
\end{aligned}
$$

Lemma 8. Assume that $c$ is a curve with $\|\dddot{c}\|<C^{\prime}$. Then

$$
\|\alpha(c(t)-c(-t))-(c(\alpha t)-c(-\alpha t))\| \leq\left|\frac{\alpha+\alpha^{3}}{3}\right| C^{\prime} t^{3} .
$$

Proof. With a Taylor expansion of degree three the left hand side in (4.11) expands to $\left\|\alpha \frac{t^{3}}{6}\left(\dddot{c}\left(\theta_{1} t\right)+\dddot{c}\left(-\theta_{2} t\right)-\alpha^{2} \dddot{c}\left(\theta_{3} \alpha t\right)-\alpha^{2} \dddot{c}\left(-\theta_{4} \alpha t\right)\right)\right\|$ with factors $\theta_{i} \in(0,1)$. This implies (4.11).

4.5. Auxiliary inequalities concerning geodesic subdivision. It has been shown in [22] that a linear affinely invariant subdivision scheme with finite mask and its analogous geodesic scheme fulfill the inequality (2.20), both for a surface and for a matrix group of constant velocity. In this paper we are concerned with first order proximity conditions, i.e., especially with verification of (2.22).

We consider a surface $M$ contained in the Euclidean vector space $\mathbb{R}^{n}$, which is equipped with geodesics - either in the sense of elementary differential geometry, or in the matrix group sense. In both cases, geodesics are the solution curves of a differential equation of the form

$$
\ddot{c}(t)=B_{c(t)}(\dot{c}(t), \dot{c}(t))
$$

where $B_{x}$ is either the second fundamental form of (4.2) or the expression defined by (4.6). $B_{x}$ is supposed to depend smoothly on the point $x$. This is trivial for the group case, and follows from $C^{3}$ smoothness of the surface under consideration in the Riemannian case. Recall that $B_{x}$ in both cases is symmetric and bilinear, and that solution curves are traversed with constant velocity. We use the orthogonal projection $\pi_{x}$ onto the tangent space $T_{x} M$ in order to extend the definition of $B_{x}$ of (4.12) to arguments not necessarily tangent to the surface $M$ :

$$
B_{x}(v, w):=B_{x}\left(\pi_{x}(v), \pi_{x}(w)\right) .
$$

Thus $B_{x}$ becomes an $n \times n$ matrix smoothly dependent on $x$. Differentiation of $B_{x}$ is understood component-wise. This way of extending the second fundamental 
form from the tangent space of the surface under consideration to entire ambient space has been used for averaging e.g. in [21].

We consider such open subsets $U$ of $M$ where there exist constants $D, D^{\prime}$ with the property that

$$
\begin{aligned}
& x \in U, \quad u, v, w \in T_{x} M, \quad\|u\|,\|v\|,\|w\| \leq 1 \\
\Longrightarrow \quad & \left\|B_{x}(v, w)\right\| \leq D, \quad\left\|\left(\partial_{u} B\right)_{x}(v, w)\right\| \leq D^{\prime} .
\end{aligned}
$$

Clearly $D, D^{\prime}$ exist locally in $M$, and globally if $M$ is compact. In the surface case the existence of global constants $D$ and $D^{\prime}$ means that normal curvatures and change of normal curvatures, respectively, are bounded. In the case of a matrix group of constant velocity, it is shown in [22] that there is a global constant $D$, which can be chosen as any $D$ with the property that for all $v, w \in \mathbb{R}^{n \times n}$,

$$
\|v w\| \leq D\|v\|\|w\| \text {. }
$$

Lemma 9. If $c(t)=\exp _{p}(t v)$ with $\|\dot{c}\|=1$, then with $D$ from (4.14),

$$
\|\ddot{c}\| \leq D \text {. }
$$

The proof is easy and can be found in [22]. The following result analogously estimates the norm of the third derivative of geodesics:

Lemma 10. If $c(t)=\exp _{p}(t v)$ with $\|\dot{c}\|=1$, then $\|\dddot{c}\|$ locally has an upper bound $D^{\prime \prime}$, which in general is given by

$$
D^{\prime \prime}:=D^{\prime}+2 D^{2}
$$

Proof. We differentiate (4.12) and get

$$
\dddot{c}=\left(\partial_{\dot{c}} B\right)(\dot{c}, \dot{c})+2 B_{c}(\ddot{c}, \dot{c})=\left(\partial_{\dot{c}} B\right)(\dot{c}, \dot{c})+2 B_{c}\left(B_{c}(\dot{c}, \dot{c}), \dot{c}\right),
$$

With $D, D^{\prime}$ from $(4.14)$, (4.17) follows immediately.

Remark: For geodesics in matrix groups which have the property that left translations $x \mapsto g x$ are isometric with respect to some Euclidean metric in the space $\mathbb{R}^{n \times n}$ of matrices, we show that there is a global constant $D^{\prime \prime}$. The Euclidean motion group falls within this category [22]. The geodesic is of the form $c(t)=g \exp (t v)$, for some matrix $v$ tangent to the given group. Then $\dot{c}(t)=g \exp (t v) v=c(t) v$ and $\dddot{c}(t)=g \exp (t v) v^{3}=c(t) v^{3}$. By left invariance of the metric, we have $\|\dot{c}(t)\|=\|v\|$. It was assumed that geodesics are traversed with unit velocity, so $\|v\|=1$. It follows that $\|\dddot{c}\|=\left\|c(t) v^{3}\right\|=\left\|v^{3}\right\| \leq$ $D\left\|v^{2}\right\| \cdot\|v\| \leq D^{2}\|v\|^{3} \leq D^{2}$, with $D$ from (4.15).

Lemma 11. Assume that (4.14) holds true with $D>0$ and an open set $U$, and that the points $x, y$ are joined by a unique shortest geodesic of length $\leq 1 / D$. If the geodesic segment used in $g$-av $v_{\alpha}(x, y)$ is contained in $U$, then with $\beta=1-\alpha$, we have

$$
\left\|\operatorname{av}_{\alpha}(x, y)-g-a v_{\alpha}(x, y)\right\| \leq 2 D \min \left(|\alpha|+\alpha^{2},|\beta|+\beta^{2}\right)\|x-y\|^{2} .
$$




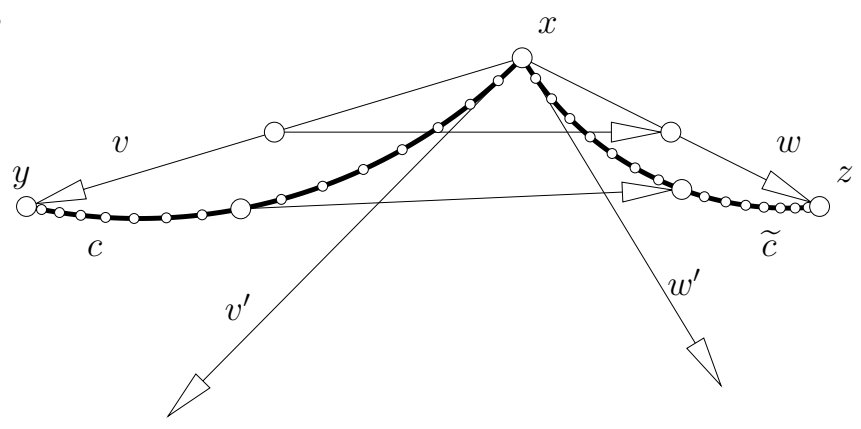

FiguRE 2. Illustration of the proof of Lemma 14.

The proofs of this and the following result are given in [22].

Lemma 12. Let $U$ and $D$ be as in (4.14). Consider an affinely invariant subdivision scheme $S$ with finite mask and its analogous geodesic scheme $T$. Let the class $\mathcal{P}_{U, \delta}^{\prime}$ consist of all polygons $p$ in $U$ with $d(p)<\delta$ and which have the property that all geodesic segments used in subdividing according to $T$ are contained in $U$.

Then $S$ and $T$ fulfill (2.20) for all polygons $p \in \mathcal{P}_{U, \delta}^{\prime}$. The constant $C$ in (2.20) depends on $T, D$, and $\delta$.

Lemma 14 below is the basis for comparing difference sequences of sequences generated by linear and by geodesic subdivision. Its proof makes use of

Lemma 13. Assume that $D$ is chosen such that (4.14) holds (i.e., $\|B\| \leq D$ ), that $\left\|v-v^{\prime}\right\|,\left\|w-w^{\prime}\right\| \leq d$, and that $\|v\| \leq \varepsilon_{1},\|w\| \leq \varepsilon_{2}$. Then $\| B(v+w, v-$ $w)-B\left(v^{\prime}+w^{\prime}, v^{\prime}-w^{\prime}\right) \| \leq 4 D d\left(\varepsilon_{1}+\varepsilon_{2}+d\right)$.

Proof. We have $v^{\prime}+w^{\prime}=v+w+r$ and $v^{\prime}-w^{\prime}=v-w+s$ with $\|r\|,\|s\| \leq 2 d$. It follows that $B\left(v^{\prime}+w^{\prime}, v^{\prime}-w^{\prime}\right)=B(v+w, v-w)+B(v+w, s)+B(r, v-w)+B(r, s)$. Thus $\left\|B(v+w, v-w)-B\left(v^{\prime}+w^{\prime}, v^{\prime}-w^{\prime}\right)\right\|$ is bounded from above by $\| B(v+$ $w, s)\|+\| B(r, v-w)\|+\| B(r, s) \| \leq D \cdot\left(\varepsilon_{1}+\varepsilon_{2}\right) \cdot 2 d+D \cdot 2 d \cdot\left(\varepsilon_{1}+\varepsilon_{2}\right)+D \cdot 2 d \cdot 2 d$.

Lemma 14. Assume that $\alpha, \beta \in \mathbb{R}$ have the property that either

$$
\alpha=\beta \text { or } \alpha=1-\beta \text {. }
$$

Then locally there are constants $C, \delta>0$, depending on $\alpha, \beta$, on the constants $D$ of (4.14) and $D^{\prime \prime}$ of Lemma 10, such that whenever the geodesic distances of the three points $x, y=x+v, z=x+w$ are smaller than $\delta$, we have the estimate

$$
\begin{aligned}
h & =g-a v_{\alpha}(x, y)-g-a v_{\beta}(x, z)-\operatorname{av}_{\alpha}(x, y)+\operatorname{av}_{\beta}(x, z), \\
& \left.\Longrightarrow\|h\| \leq C\left[(\|v\|+\|w\|) \cdot\|v-w\|+\|v\|^{3}+\|w\|^{3}\right)\right] .
\end{aligned}
$$

Proof. The geodesics used in averaging are denoted by $c$ and $\widetilde{c}: \widetilde{c}(0)=c(0)=x$, $c(\tau)=y, \widetilde{c}(\sigma)=z$. We use a Taylor expansion with a remainder term of degree 
3:

$$
\begin{aligned}
h & =c(\alpha \tau)-\widetilde{c}(\beta \sigma)-((1-\alpha) x+\alpha c(\tau))+((1-\beta) x+\beta \widetilde{c}(\sigma)) \\
& \left.=\frac{\alpha^{2}-\alpha}{2} \tau^{2} \ddot{c}(0)-\frac{\beta^{2}-\beta}{2} \sigma^{2} \ddot{\widetilde{c}}(0)\right) \\
& +\frac{1}{3 !}\left(\alpha^{3} \tau^{3} \dddot{c}\left(\theta_{1} \tau\right)-\alpha \tau^{3} \dddot{c}\left(\theta_{2} \alpha \tau\right)\right)-\frac{1}{3 !}(\ldots),
\end{aligned}
$$

where $0 \leq \theta_{1}, \ldots, \theta_{4} \leq 1$. We denote the second degree and third degree terms on the right hand side of the above equation by " $(* *)$ " and " $(* * *)$ ", respectively. Symmetric bilinear mappings $B$ have the property that $B(a, a)-B(b, b)=B(a-$ $b, a+b)$. We compute

$$
\begin{aligned}
& v^{\prime}:=\tau \dot{c}(0), w^{\prime}:=\sigma \dot{\widetilde{c}}(0) \Longrightarrow \tau^{2} \ddot{c}(0)-\sigma^{2} \ddot{\widetilde{c}}(0)= \\
& =B_{x}\left(v^{\prime}, v^{\prime}\right)-B_{x}\left(w^{\prime}, w^{\prime}\right)=B_{x}\left(v^{\prime}+w^{\prime}, v^{\prime}-w^{\prime}\right) .
\end{aligned}
$$

We want to apply Lemma 7 to the curves $c$ and $\widetilde{c}$ in order to get estimates for $\|\tau \dot{c}(0)-v\|$ and $\|\sigma \dot{\widetilde{c}}(0)-w\|$. As both $c$ and $\widetilde{c}$ are geodesics and traversed by unit velocity, the geodesic distance of the point $c(0)$ and $c(t)$ equals $t$, and the same for $\widetilde{c}$. According to Lemma 7 this means that by choosing $\delta<1 / D$ we can achieve that

$$
|\tau|<2\|v\|,|\sigma|<2\|w\|,\left\|v^{\prime}-v\right\| \leq \frac{D \tau^{2}}{2},\left\|w^{\prime}-w\right\| \leq \frac{D \sigma^{2}}{2}
$$

(from left to right, this is (4.10), (4.10), (4.9), and (4.9)). The expression in (4.20) is now estimated by means of Lemma 13, with $d=2 D \max (\|v\|,\|w\|)^{2}$, $\varepsilon_{1}=\|v\|, \varepsilon_{2}=\|w\|:$

$$
\left\|\tau^{2} \ddot{c}(0)-\sigma^{2} \ddot{\widetilde{c}}(0)\right\| \leq\|B(v+w, v-w)\|+4 D d\left(\varepsilon_{1}+\varepsilon_{2}+d\right) .
$$

By our assumption, $\alpha^{2}-\alpha=\beta^{2}-\beta$. It follows that there is a constant $C^{\prime}$, dependent on $D, \alpha, \beta$ and $\delta$ such that

$$
(* *) \leq\left|\frac{\alpha^{2}-\alpha}{2}\right| D\|v-w\|(\|v\|+\|w\|)+C^{\prime} \max (\|v\|,\|w\|)^{3} .
$$

As to $(* * *)$, we use $(4.21)$ to conclude that

$$
(* * *) \leq \frac{8 D^{\prime \prime}}{3} \max (|1+\alpha|,|1+\beta|)\left|\alpha^{2}-\alpha\right|\left(\|v\|^{3}+\|w\|^{3}\right) .
$$

By combining the estimates for the second and the third degree terms, we get (4.19) and the proof is complete.

4.6. Proximity conditions for geodesic B-spline schemes. We now consider B-spline subdivision $S=S_{(3)}$ according to $(2.2)$. $S_{(3)}$ is the first B-spline scheme which is $C^{2}$. From the proximity inequality for $S_{(3)}$ we will get proximity inequalities for a wide class of schemes, which is described by Def. 9 below. Let $T$ be the geodesic analogue of $S_{(3)}$. In the first three rounds of the recursion in (2.2), geodesic averaging takes place within the same geodesic lines which connect 


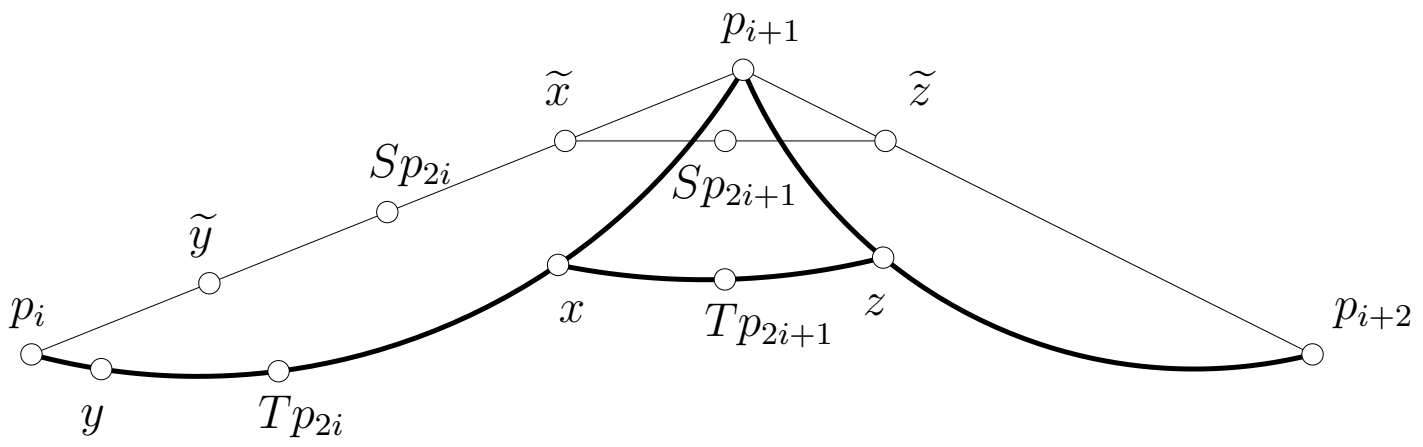

FiguRE 3. Illustration of the proof of Lemma 15.

the points of the original polygon $p$. It follows that the geodesic analogue of $S_{(3)}$ defined by the recursion (2.2) has the explicit representation

$$
\begin{aligned}
T p_{2 i} & =g-\operatorname{av}_{1 / 2}\left(p_{i}, p_{i+1}\right), \\
T p_{2 i+1} & =g-\operatorname{av}_{1 / 2}\left(g-\operatorname{av}_{1 / 4}\left(p_{i+1}, p_{i}\right), \operatorname{g}-\operatorname{av}_{1 / 4}\left(p_{i+1}, p_{i+2}\right)\right) ;
\end{aligned}
$$

and of course for $S_{(3)}$ we have

$$
\begin{aligned}
S_{(3)} p_{2 i} & =\operatorname{av}_{1 / 2}\left(p_{i}, p_{i+1}\right) \\
S_{(3)} p_{2 i+1} & =\operatorname{av}_{1 / 2}\left(\operatorname{av}_{1 / 4}\left(p_{i+1}, p_{i}\right), \operatorname{av}_{1 / 4}\left(p_{i+1}, p_{i+2}\right)\right) .
\end{aligned}
$$

Lemma 15. Consider the subdivision scheme $S=S_{(3)}$ defined by (2.2) (the cubic $B$-spline scheme), and its analogous geodesic scheme $T$. Then for all open sets $U$ where there exist constants $D$ and $D^{\prime \prime}$ according to (4.14) and Lemma 10, there is $\delta>0$ such that that $S$ and $T$ fulfill (2.22) for $p \in \mathcal{P}_{U, \delta}$.

Proof. We employ the definition of $T$ given by (4.25). $\Delta T p_{i}$ is computed differently depending on whether $T p_{i}$ is on an edge of the original polygon or not. We consider only one of these two cases, because changing the sense of direction in $p$ exchanges these two cases, and $T$ is invariant with respect to this transformation.

We use geodesics $c_{i}$ with $c_{i}(0)=p_{i}, c_{i}\left(\tau_{i}\right)=p_{i+1}$, and also the geodesics $\bar{c}$ which parametrize the same curve segment the other way round, i.e., $\bar{c}_{i}(0)=p_{i+1}$, $\bar{c}_{i}\left(\tau_{i}\right)=p_{i}$. Let $\alpha=1 / 4$ and introduce the points $x, y, z, \widetilde{x}, \widetilde{y}, \widetilde{z}$ :

$$
\begin{aligned}
& x=\operatorname{g-av}_{\alpha}\left(p_{i+1}, p_{i}\right), y=\operatorname{g-av}\left(p_{i}, p_{i+1}\right), z=g-\operatorname{av}_{\alpha}\left(p_{i+1}, p_{i+2}\right), \\
& \widetilde{x}=\operatorname{av}_{\alpha}\left(p_{i+1}, p_{i}\right), \widetilde{y}=\operatorname{av}_{\alpha}\left(p_{i}, p_{i+1}\right), \widetilde{z}=\operatorname{av}_{\alpha}\left(p_{i+1}, p_{i+2}\right) .
\end{aligned}
$$

It follows that

$$
\begin{aligned}
& S p_{2 i}=\operatorname{av}_{1 / 2}(\widetilde{x}, \widetilde{y}), S p_{2 i+1}=\operatorname{av}_{1 / 2}(\widetilde{x}, \widetilde{z}), \\
& T p_{2 i}=\mathrm{g}_{-\mathrm{av}_{1 / 2}}(x, y), T p_{2 i+1}=\mathrm{g}-\mathrm{av}_{1 / 2}(x, z) .
\end{aligned}
$$


We use the triangle inequality to split $\left\|\Delta S p_{2 i}-\Delta T p_{2 i}\right\|$ into two parts:

$$
\begin{aligned}
& \left\|\Delta S p_{2 i}-\Delta T p_{2 i}\right\|=\left\|S p_{2 i+1}-S p_{2 i}-T p_{2 i+1}+T p_{2 i}\right\| \\
= & \left\|\operatorname{av}_{1 / 2}(\widetilde{x}, \widetilde{z})-\operatorname{av}_{1 / 2}(\widetilde{x}, \widetilde{y})-g-\operatorname{av}_{1 / 2}(x, z)+g-\operatorname{av}_{1 / 2}(x, y)\right\| \\
\leq & \left\|\operatorname{av}_{1 / 2}(x, z)-\operatorname{av}_{1 / 2}(x, y)-g-\operatorname{av}_{1 / 2}(x, z)+g-\operatorname{av}_{1 / 2}(x, y)\right\| \\
+ & \left\|\operatorname{av}_{1 / 2}(\widetilde{x}, \widetilde{z})-\operatorname{av}_{1 / 2}(\widetilde{x}, \widetilde{y})-\operatorname{av}_{1 / 2}(x, z)+\operatorname{av}_{1 / 2}(x, y)\right\|
\end{aligned}
$$

Lemma 14 with $\alpha=\beta=1 / 2$ implies the inequality

$$
\begin{aligned}
(*) & \leq C[(\|y-x\|+\|z-x\|)\|(y-x)-(x-z)\| \\
& \left.+\|y-x\|^{3}+\|z-x\|^{3}\right] .
\end{aligned}
$$

By Lemma 12, $S$ and $T$ fulfill (2.20). This implies that each of the three vectors

$$
(x-y)-\Delta p_{i} / 2,\left(x-p_{i+1}\right)-\left(-\Delta p_{i}\right) / 4,\left(z-p_{i+1}\right)-\Delta p_{i+1} / 4,
$$

is bounded by a constant times $d(p)^{2}$. It follows that there is a constant $C^{\prime}$ such that

$$
\begin{aligned}
& \left\|[(y-x)-(x-z)]-\left[\left(-\Delta p_{i} / 2\right)-\left(-\Delta p_{i} / 4-\Delta p_{i+1} / 4\right)\right]\right\| \\
= & \left\|[(y-x)-(x-z)]-\frac{1}{4} \Delta^{2} p_{i}\right\| \leq C^{\prime} d(p)^{2},
\end{aligned}
$$

which implies that

$$
\|(y-x)-(x-z)\| \leq C^{\prime} d(p)^{2}+d(\Delta p) / 4 .
$$

Consequently (4.32) implies that there is a constant $C^{\prime \prime}$ such that

$$
\left.(*) \leq C^{\prime \prime}\left[d(p) d(\Delta p)+d(p)^{3}\right)\right] .
$$

Lemma 14 with $\alpha=\beta=1 / 4$ and the points $p_{i+1}, p_{i}$ and $p_{i+2}$ yields

$$
\begin{aligned}
\|(x-z)-(\widetilde{x}-\widetilde{z})\| & \leq \bar{C}\left[\left(\left\|\Delta p_{i}\right\|+\left\|\Delta p_{i+1}\right\|\right) \cdot\left\|\Delta^{2} p_{i}\right\|\right. \\
& \left.\left.+\left\|\Delta p_{i}\right\|^{3}+\left\|\Delta p_{i+1}\right\|^{3}\right)\right] .
\end{aligned}
$$

In order to estimate $(x-y)-(\widetilde{x}-\widetilde{y})$, we use the geodesic $c$ defined by $c(t)=$ $c_{i}\left(t+\tau_{i} / 2\right)$. We note that $\dddot{c}$ is bounded according to (4.17) and appeal to (4.11):

$$
\begin{aligned}
\|(x-y)-(\widetilde{x}-\widetilde{y})\| & =\|\left(c\left(\tau_{i} / 4\right)-c\left(-\tau_{i} / 4\right)\right) \\
& -\frac{1}{2}\left(c\left(\tau_{i} / 2\right)-c\left(-\tau_{i} / 2\right)\right) \| \leq \bar{C} \tau_{i}^{3} .
\end{aligned}
$$

By Lemma $7, \tau_{i} \leq 2\left\|\Delta p_{i}\right\|$, if the geodesic distance of the points $p_{i}$ and $p_{i+1}$ is bounded appropriately. Thus we get

$$
\begin{aligned}
(* *) & \leq \frac{1}{2}(\|(y-x)-(\widetilde{y}-\widetilde{x})\|+\|(x-z)-(\widetilde{x}-\widetilde{z})\|) \\
& \leq C^{\prime \prime \prime \prime}\left(d(p)^{3}+d(p) d(\Delta p)\right) .
\end{aligned}
$$

The two estimates for $(*)$ and $(* *)$ together show (2.22) for $S$ and $T$. 
4.7. Proximity inequalities and geodesic averages. After considering the subdivision rule $S_{(3)}$ above, this subsection is a further step towards our aim of showing proximity inequalities of a wider class of subdivision rules. It is concerned with schemes $S, T$, which arise from schemes $\widetilde{S}, \widetilde{T}$ by adding one further round of averaging to each.

Lemma 16. Assume that $\widetilde{S}$ and $\widetilde{T}$ are subdivision schemes which meet (2.22). Suppose that $\widetilde{S}$ has derived schemes $\widetilde{S}_{1}$ and $\widetilde{S}_{2}$. Define $S, T$ by one further step of averaging:

$$
S p_{i}=\operatorname{av}_{\alpha}\left(\widetilde{S} p_{i}, \widetilde{S} p_{i+1}\right), \quad T p_{i}=g-a v_{\alpha}\left(\widetilde{T} p_{i}, \widetilde{T} p_{i+1}\right),
$$

Then for all open sets $U$ where there exist constants $D$ and $D^{\prime \prime}$ according to (4.14) and Lemma 10, resp., there is $\delta>0$ such that $S$ and $T$ fulfill (2.22) for all $p \in \mathcal{P}_{U, \delta}$.

Proof. We introduce the points

$$
q_{i}=\operatorname{av}_{\alpha}\left(\widetilde{T} p_{i}, \widetilde{T} p_{i+1}\right) .
$$

It follows directly from Lemma 14 with $\beta=1-\alpha$ that there is a constant $C^{\prime}$ such that for all $p$ with $d(\widetilde{T} p)$ small enough we have

$$
\left\|\Delta q_{i}-\Delta T p_{i}\right\| \leq C^{\prime}\left(d(\widetilde{T} p) d(\Delta \widetilde{T} p)+d(\widetilde{T} p)^{3}\right) .
$$

We want to express the bound in terms of $p$ rather than $\widetilde{T} p$. We are going to use that $\widetilde{S}$ and $\widetilde{T}$ obey both $(2.20)$ and $(2.22)$, and that $\widetilde{S}$ has derived schemes $\widetilde{S}_{1}$ and $\widetilde{S}_{2}$, whence $d(\widetilde{S} p) \leq \widetilde{\mu}_{0}, d(\Delta \widetilde{S} p) \leq \widetilde{\mu}_{1} d(\Delta p)$.

$$
\begin{aligned}
d(\widetilde{T} p) & \leq d(\widetilde{S} p)+2\|\widetilde{T} p-\widetilde{S} p\| \leq \widetilde{\mu}_{0} d(p)+2 C d(p)^{2} \\
d(\Delta \widetilde{T} p) & \leq d(\Delta \widetilde{S} p)+2\|\Delta \widetilde{T} p-\Delta \widetilde{S} p\| \\
& \leq \widetilde{\mu}_{1} d(\Delta p)+2 C\left(d(p) d(\Delta p)+d(p)^{3}\right) .
\end{aligned}
$$

Inserting this in (4.41) yields an upper bound which is a polynomial in $d(p)$ and $d(\Delta p)$. Via $d(p) \leq \delta$ and therefore $d(p)^{k} \leq d(p) \delta^{k-1}$ we get

$$
\left\|\Delta q_{i}-\Delta T p_{i}\right\| \leq C^{\prime \prime}\left(d(p) d(\Delta p)+d(p)^{3}\right) .
$$

Further,

$$
\begin{aligned}
\left\|\Delta S p_{i}-\Delta q_{i}\right\| & =\left\|(1-\alpha) \Delta \widetilde{S} p_{i}+\alpha \Delta \widetilde{S} p_{i+1}-(1-\alpha) \Delta \widetilde{T} p_{i}-\alpha \Delta \widetilde{T} p_{i+1}\right\| \\
& \leq(|1-\alpha|+|\alpha|)\|\Delta \widetilde{S} p-\Delta \widetilde{T} p\| .
\end{aligned}
$$

We use (4.41) and (2.22) to show that

$$
\begin{aligned}
& \left\|\Delta S p_{i}-\Delta T p_{i}\right\| \leq\left\|\Delta S p_{i}-\Delta q_{i}\right\|+\left\|\Delta q_{i}-\Delta T p_{i}\right\| \\
& \leq\left((|1-\alpha|+|\alpha|) C+C^{\prime \prime}\right)\left(d(p) d(\Delta p)+d(p)^{3}\right) .
\end{aligned}
$$

The proof is complete. 
4.8. Proximity for projection subdivision. The part of this paper concerning projection subdivision is shorter than the analogous part on geodesic subdivision. The reason for this is that the proofs are analogous, once some basic inequalities are established.

We require the existence of upper bounds for the norms of the projection's derivatives. In compact subsets, upper bounds always exist in analogy to the constant $D$ of (4.14).

We consider an open subset $U$ of $\mathbb{R}^{n}$ (the space where the surface under consideration is contained in), where there are constants $D, D^{\prime}, D^{\prime \prime} \geq 0$ such that

$$
x \in U \Longrightarrow\left\|d_{x} P\right\| \leq D,\left\|d_{x}^{2} P\right\| \leq D^{\prime},\left\|d_{x}^{3} P\right\| \leq D^{\prime \prime} .
$$

The following is an immediate consequence of (4.44):

Lemma 17. If $c(t)=P(x+t v)$ with $\|v\|=1$, then $\|\ddot{c}\|<D^{\prime}$ and $\|\dddot{c}\|<D^{\prime \prime}$, with $D^{\prime}, D^{\prime \prime}$ from (4.44).

Lemma 18. Assume that $U, D, D^{\prime}$ are as in (4.44), and that the straight line segment which contains the points $x, y,(1-\alpha) x+\alpha y$ is contained in $U$. With $\beta=1-\alpha$,

$$
\left\|\operatorname{av}_{\alpha}(x, y)-P \operatorname{av}_{\alpha}(x, y)\right\| \leq \frac{D^{\prime}}{2} \min \left(|\alpha|+\alpha^{2},|\beta|+\beta^{2}\right)\|x-y\|^{2} .
$$

The proofs of this and the following result can be found in [22].

Lemma 19. (the projection analogue of Lemma 12) Let $U, D$, and $D^{\prime}$ be as in (4.44). Consider an affinely invariant subdivision scheme $S$ and its analogous projection scheme $T$. Let the class $\mathcal{P}_{U, \delta}^{\prime}$ consist of all surface polygons $p$ with $d(p)<\delta$, and such that the line segments used in averaging in the application of $T$ are inside $U$.

Then $S$ and $T$ fulfill (2.20) for all polygons $p \in \mathcal{P}_{U, \delta}^{\prime}$. The constant $C$ in $(2.20)$ depends on $T, D, D^{\prime}$, and $\delta$.

In order to show that (2.22) holds (which is part of the first order proximity condition), we proceed in a way analogous to the geodesic case. The next lemma is the projection variant of Lemma 14 .

Lemma 20. Choose $\alpha, \beta$ with $\alpha(1-\alpha)=\beta(1-\beta)$, and an open subset $U$ such that in $U$ exist constants $D, D^{\prime}, D^{\prime \prime}$ according to (4.44). Then there are constants $C, C^{\prime}, \delta>0$, depending on $\alpha, \beta, D, D^{\prime}, D^{\prime \prime}$, such that for all points $x, y=x+v$, $z=x+w$ the estimate

$$
\begin{aligned}
& P \operatorname{Pav}_{\alpha}(x, y)-\operatorname{Pav}_{\beta}(x, z)-\operatorname{av}_{\alpha}(x, y)+\operatorname{av}_{\beta}(x, z) \\
\leq & C(\|v\|+\|w\|) \cdot\|v-w\|+C^{\prime}\left(\|v\|^{3}+\|w\|^{3}\right),
\end{aligned}
$$

is true, provided $\|v\|,\|w\|<\delta$. 
Proof. The proof is similar to the proof of Lemma 14, but easier. We denote the combination of averages used in (4.46) by the symbol $h$ and apply Taylor's formula to the curves defined by $c(t)=P\left(x+t v_{0}\right), \widetilde{c}(t)=P\left(x-t w_{0}\right)$, where $v_{0}$ and $w_{0}$ are unit vectors parallel to $v$ and $w$, resp. We assume that $c(\tau)=y$ and $\widetilde{c}(\sigma)=z$ and get $h=c(\alpha \tau)-\widetilde{c}(\alpha \sigma)-((1-\alpha) x+\alpha c(\tau))+((1-\beta) x+\beta \widetilde{c}(\sigma))=$ $\frac{\alpha^{2}-\alpha}{2 !}\left(\tau^{2} \ddot{c}(0)-\sigma^{2} \ddot{\widetilde{c}}(0)\right)+\frac{1}{3 !}\left(\alpha^{3} \tau^{3} \dddot{c}\left(\theta_{1} \tau\right)-\alpha \tau^{3} \dddot{c}\left(\theta_{2} \alpha \tau\right)+\ldots\right)$, where $0<\theta_{i}<1$. The second degree term may be estimated as follows:

$$
\begin{aligned}
& \left\|\tau^{2} \ddot{c}(0)-\sigma^{2} \ddot{\widetilde{c}}(0)\right\|=\left\|d_{x}^{2} P(v, v)-d_{x}^{2} P(w, w)\right\| \\
& =\left\|d_{x}^{2} P(v-w, v+w)\right\| \leq D^{\prime}\|v-w\|(\|v\|+\|w\|) .
\end{aligned}
$$

The third degree remainder term above is obviously bounded by

$$
\max \left(\left|\frac{\alpha^{3}+\alpha}{3}\right|,\left|\frac{\beta^{3}+\beta}{3}\right|\right) D^{\prime \prime}\left(\|v\|^{3}+\|w\|^{3}\right),
$$

which concludes the proof.

Next we consider a projection analogue " $T$ " of $S_{(3)}$ based on the representation (4.26). It is given by

$$
\begin{aligned}
T p_{2 i} & =P \operatorname{av}_{1 / 2}\left(p_{i}, p_{i+1}\right) \\
T p_{2 i+1} & =\operatorname{Pav}_{1 / 2}\left(\operatorname{Pav}_{1 / 4}\left(p_{i+1}, p_{i}\right), \operatorname{Pav}_{1 / 4}\left(p_{i+1}, p_{i+2}\right)\right) .
\end{aligned}
$$

Note that (4.49) is not the direct projection analogue of the recursive definition (2.2). The difference is that the projection is applied after every averaging step, instead just once, after all averagings. We use (4.49) instead of the direct projection analogue because we want to treat projection subdivision in a way as analogous as possible to geodesic subdivision.

Lemma 21. Assume that $S$ is the cubic B-spline scheme $S=S_{(3)}$ defined by (4.26) and $T$ is its projection analogue (4.49). Assume that the open set $U$ is chosen as in Lemma 20. Then there is $\delta>0$ such that $S$ and $T$ fulfill (2.22) for all $p \in \mathcal{P}_{U, \delta}$.

Proof. We turn the proof of Lemma 15 into a proof of this result, if we make the following replacements: (i) g-av $\rightsquigarrow P$ av; (ii) references to Lemma $14 \rightsquigarrow$ references to Lemma 20; (iii) with $\tau_{i}=\left\|\Delta p_{i}\right\|$, we let $c_{i}(t)=P\left(p_{i}+t \Delta p_{i} / \tau_{i}\right), \bar{c}_{i}(t)=$ $c_{i}\left(\tau_{i}-t\right)$, and $c(t)=c\left(t+\tau_{i} / 2\right)$; (iv) an upper bound for $\dddot{c}$ is furnished not by (4.17), but by Lemma 17; $(v)$ we may use $\tau_{i}=\left\|\Delta p_{i}\right\|$ instead of appealing to Lemma 7 in order to get an upper bound for $\tau_{i}$ in terms of $\left\|\Delta p_{i}\right\|$.

Lemma 22. Assume that $\widetilde{S}$ and $\widetilde{T}$ are subdivision schemes which meet the inequality (2.22). Suppose that $\widetilde{S}$ has derived schemes $\widetilde{S}_{1}, \widetilde{S}_{2}$. Define $S, T$ by one further step of averaging:

$$
S p_{i}=\operatorname{av}_{\alpha}\left(\widetilde{S} p_{i}, \widetilde{S} p_{i+1}\right), \quad T p_{i}=P \operatorname{Pav}_{\alpha}\left(\widetilde{T} p_{i}, \widetilde{T} p_{i+1}\right),
$$

If $U$ is chosen as in Lemma 20, there is $\delta>0$ such that that $S$ and $T$ fulfill (2.22) for all $p \in \mathcal{P}_{U, \delta}$. 
Proof. We turn the proof of Lemma 16 into a proof of Lemma 22 by making the same substitutions as in the proof of Lemma 21.

Before the start of the next section, let us summarize what was achieved in Section 4: We have defined geodesic schemes, geodesic-like schemes in matrix groups of constant velocity, and projection schemes. We repeated some known properties concerning zero order proximity. The major part of this section was to establish the inequality (2.22) for certain nonlinear analogues of certain linear schemes, thus verifying in part the necessary conditions of Th. 5. The next subsection collects the results obtained so far, and summarizes them in a few theorems.

\section{5. $C^{2}$ Smoothness of FaCtorizable Subdivision Rules}

5.1. Factorizable subdivision rules. The lemmas above do not so easily allow results concerning $C^{2}$ smoothness of limit curves which are as general as the results in [22]. Until now, we have shown that cubic B-spline subdivision and its nonlinear analogues meet the inequality (2.22), and we have also shown that once such a property is established, further rounds of averaging do not destroy it. It follows that we can prove smoothness results for the following class of subdivision schemes:

Definition 9. The class "F" of subdivision rules is generated as follows:

1. The cubic B-spline scheme $S_{(3)} p$ is in $F$.

2. If $S \in F$, then $S^{m} \in F$ for all integers $m>0$.

3. If $S \in F$ and $A_{\alpha}$ is the averaging operator $\left(A_{\alpha} p\right)_{j}:=\operatorname{av}_{\alpha}\left(p_{j}, p_{j+1}\right)$, then $A_{\alpha} S \in F$.

4. If $S \in F$ and $\sigma_{j}$ is the shift operator $\left(\sigma_{j} p\right)_{i}:=p_{i-j}$, then $\sigma_{j} S \in F$.

Applying one round of averaging with factor $\alpha$ to the polygon $p$ is a "subdivision" process $A_{\alpha}$ with dilation factor $N=1$. It has the symbol

$$
a_{\alpha}(z)=\left((1-\alpha)+\alpha z^{-1}\right)=\frac{1-\alpha}{z}\left(z-\frac{\alpha}{\alpha-1}\right) .
$$

It immediately follows that any affinely invariant subdivision rule with symbol

$$
a(z)=C z^{m}(1+z)^{4}\left(z-\beta_{1}\right) \cdots\left(z-\beta_{n}\right), \quad m \in \mathbb{Z}, C \in \mathbb{R}, \beta_{i} \neq 1
$$

is contained in the class $F$. Especially all the B-spline schemes $S_{(n)}$ with $n \geq 3$ are.

Any subdivision rule $S$ in the class " $F$ " is defined by taking the cubic B-spline scheme and applying a finite number of the operations described in Def. 9 to it. This leads to

Definition 10. The canonical representation of the linear scheme $S \in F$ in terms of averages is defined recursively in terms of the steps in Def. 9:

1. If $S=S_{(3)}$ p, use (4.26) to represent $S$ in terms of averages. 
2. If $S=\bar{S}^{m}$, apply the representation of $\bar{S}$ in terms of averages $m$ times. Thus if $\bar{T}$ is the analogue of $\bar{S}, \bar{T}^{m}$ is the analogue of $\bar{S}^{m}$.

3. If $S=A_{\alpha} \bar{S}$, add the averaging step av $a_{\alpha}$ to the representation of $\bar{S}$.

4. If $S=\sigma_{j} \bar{S}$, shift the indices in $\bar{S}$ 's representation in order to get a representation of $S$.

As has already been mentioned, the B-spline schemes of degree $n \geq 3$ are contained in the class " $F$ ". In general, the interpolatory Dubuc-Deslauriers schemes [5] are not, but it has been shown in [22] that the four-point scheme with weight $1 / 16$ of [10] has this property. The $C^{2}$ four-point scheme of [9] with weight $w>0$ has the symbol

$$
\left.a(z)=\frac{1}{4}(1+z)^{3} z^{-1}\left(1+4 w\left(-5\left(z^{2}+\frac{1}{z^{2}}\right)+8\left(z+\frac{1}{z}\right)-6\right)\right)\right) .
$$

For $w=1 / 128$, this scheme is contained in the class " $F$ ".

Lemma 23. If $S$ is a subdivision scheme in the class " $F$ ", represented in terms of averages in the canonical way of Def. 10, and $T$ is its analogous geodesic or projection scheme, then $S$ and $T$ obey (2.22) for all $p$ with $d(p)$ small enough.

Proof. This is shown by recursion over the construction of $S$. The numbers refer to Def. 9 and Def. 10.

1. If $S=S_{(3)}$, the result consists of Lemma 15 and Lemma 21 .

2. If the result applies to $\bar{S}$ and $\bar{T}$, then Lemma 3 shows that it applies to $S=\bar{S}^{m}$ and $T=\bar{T}^{m}$ also.

3. All schemes constructed iteratively have the property that their first and second derived schemes exist. If $S=A_{\alpha} \bar{S}$, and the result is true for $\bar{S}$, then Lemma 16 and Lemma 22 show that it is true also for $S$.

4. An index shift is irrelevant for proximity.

Our aim is a result on the $C^{2}$ smoothness of nonlinear schemes " $T$ " analogous to schemes " $S$ " of class " $F$ " (Th. 7 below). For that, we need the general proximity condition of Def. 4. The previous result establishes its hard part, namely (2.22). It quantifies the error we make when constructing a scheme $T$ analogous to $S$. The remaining part, Equ. (2.23) refers to properties of $S$ and will be dealt with in the next subsection.

5.2. Subdivision in Surfaces, Lie groups, and Riemannian manifolds. This final subsection combines the previous results concerning subdivision on surfaces and matrix groups, and also extends them to Riemannian manifolds and abstract Lie groups. We give a small extra definition, which collects properties of subdivision schemes which our results apply to.

Note that for all schemes in the class " $F$ ", the derived schemes $S_{1}, S_{1}, S_{3}$ exist, because it does for the B-spline scheme $S_{(3)}$, and none of the operations described by Def. 9 destroy this property. It follows that the factors $\mu_{0}, \mu_{1}, \mu_{2}$ according to (2.15) exist. 
Definition 11. A scheme $S$ with dilation factor $N$ is called 2-admissible, if it is in the class " $F$ ", if there is an iterate $S^{m}$, such that the coefficients $\widetilde{\mu}_{i}=\frac{1}{N^{m}}\left\|S_{i+1}^{m}\right\|$ (which correspond to the smoothness condition of $S^{m}$ ) obey the inequalities

$$
\widetilde{\mu}_{0}^{3}<1 / N^{2 m}, \quad \widetilde{\mu}_{0} \widetilde{\mu}_{1}<1 / N^{m}, \quad \widetilde{\mu}_{2}<1 .
$$

Lemma 24. If a scheme $S$ is 2-admissible, then, in the notation of Def. 11, there are $\mu_{i}^{*}$ with $\widetilde{\mu}_{i}<\mu_{i}^{*}$ for $i=0,1,2$ and $\mu_{2}^{*}=1$, such that the inequalities (2.21) and (2.23) are fulfilled with the dilation factor $N^{m}$ instead of $N$.

Proof. We let $\widetilde{\mu}_{i}=\left(N^{m}\right)^{-m_{i}}$. We have $1 / N^{m} \leq \widetilde{\mu}_{i}<1$, so $0<m_{i} \leq 1$. Because of the previous inequalities, the point $\left(m_{0}, m_{1}\right)$ is a member of the planar domain defined by $m_{0}>0, m_{1}>0, m_{0} \leq 1, m_{1} \leq 1, m_{0}>2 / 3, m_{0}+m_{1}>1$, which is visualized in Fig. 1, center. It elementary that for any such point $\left(m_{0}, m_{1}\right)$, there is a point $\left(m_{0}^{*}, m_{1}^{*}\right)$ such that $m_{i}<m_{i}^{*}$ in the planar domain defined by $m_{0}^{*} \geq 0$, $m_{1}^{*} \geq 0, m_{0}^{*}<1, m_{1}^{*}<1,2 m_{0}^{*} \geq m_{1}^{*}+1, m_{0}^{*}+m_{1}^{*} \geq 1$, which is visualized in Fig. 1 , left. All points of that latter domain fulfill $m_{0}^{*} \geq 2 / 3$. It follows that there are $\mu_{i}^{*}=N^{-m_{i}^{*}}$ for $i=0,1$, such that both (2.21) and (2.23) are fulfilled.

The B-spline schemes $S_{(n)}$ for $n \geq 3$ are 2-admissible, because Def. 11 is fulfilled with $m=1, N=2, \mu_{0}=\mu_{1}=\mu_{2}=1 / 2$. The interpolatory four-point scheme of [10] is not 2-admissible, because its powers fail to fulfill a second order smoothness condition, as it is not $C^{2}$. The $C^{2}$ four-point scheme with weight $w$ of [9], whose symbol is given by (5.3), is 2-admissible for $w=1 / 128$, because then $S$ is in the class $F$ (as mentioned above), and $S^{2}$ has $\widetilde{\mu}_{2}<1, \widetilde{\mu}_{0} \widetilde{\mu}_{1}<1 / 4$, and $\widetilde{\mu}_{0}<4^{-2 / 3}$.

We are ready to state our results concerning the $C^{2}$ smoothness of subdivision in surfaces, which are an extension of the $C^{1}$ results of [22].

Theorem 7. Assume that $S$ is a 2-admissible scheme represented in terms of averages in the canonical way of Def. 10, and $T$ is its analogous geodesic scheme, where "geodesic" is understood in the surface or in the matrix group sense. If $T^{l} p(l \rightarrow \infty)$ converges to a limit curve $T^{\infty} f$, then this limit curve is $C^{2}$.

Proof. As $S$ has finite mask and convergence of $T$ is assumed, we have $d\left(T^{l} p\right) \rightarrow 0$ locally. By [22], $S$ and $T$ fulfill (2.20), and by Lemma 23, they fulfill (2.22). In view of Lemma 3, so do $S^{n}$ and $T^{n}$ for any $n$. The assumption of 2 -admissibility together with Lemma 24 now shows that there is $m>0$ such that $S^{m}, T^{m}$ fulfill the proximity conditions of Def. 4 . The derived schemes $\left(S^{m}\right)_{i}=\left(S_{i}\right)^{m}$ exist by assumption for $i=1,2,3$, so we are able to refer to Th. 6 and conclude that $T^{\infty} p=\left(T^{m}\right)^{\infty} p$ is a $C^{2}$ curve.

These results can be extended to abstract Riemannian manifolds and to a certain class of abstract Lie groups by using Nash's embedding theorem for Riemannian manifolds [15] and by considering Lie groups which are locally isomorphic to matrix Lie groups of constant velocity. The proofs are completely analogous to the proofs in [22], where results are extended to these two abstract settings. 
Corollary 1. Theorem 7 applies to geodesic subdivision in Riemannian manifolds.

In order to extend Th. 7 to abstract Lie groups, we repeat the following definition of [22]:

Definition 12. A Lie group is called of constant velocity, if it is locally isomorphic to a matrix Lie group of constant velocity.

See [19] for an introduction into Lie groups and the concept of local isomorphism of groups, which is the same as isomorphism of the corresponding Lie algebras.

Corollary 2. Theorem 7 holds in Lie groups of constant velocity.

As to projection subdivision, the following is an analogue of Th. 7. The proof is exactly the same.

Theorem 8. Assume that $S$ is a 2-admissible scheme represented in terms of averages in the canonical way, and $T$ its analogous projection scheme. If $T^{l} p$ $(l \rightarrow \infty)$ converges to a limit curve $T^{\infty} f$, then this limit curve is $C^{2}$.

\section{ACKNOWLEDGEMENTS}

The author wishes to express his thanks to Helmut Pottmann and Adi Levin for contributing ideas to this work, and especially to Nira Dyn for extensive discussions and continuing support throughout the work on this paper.

\section{REFERENCES}

[1] C. Belta and V. Kumar. An SVD-based projection method for interpolation on SE(3). IEEE Trans. Robotics Automation, 18:334-345, 2002.

[2] O. Bottema and B. Roth. Theoretical Kinematics. North-Holland, Amsterdam, 1979.

[3] Alfred S. Cavaretta, Wolfgang Dahmen, and Charles A. Micchelli. Stationary subdivision, volume 93 of Memoirs of the AMS. American Mathematical Society, 1991.

[4] I. Daubechies, O. Runborg, and W. Sweldens. Normal multiresolution approximation of curves. Constr. Approx., 20:399-463, 2004.

[5] G. Deslauriers and S. Dubuc. Symmetric iterative interpolation processes. Constr. Approx., 5:49-68, 1986.

[6] M. P. do Carmo. Riemannian Geometry. Birkhäuser Verlag, Boston Basel Berlin, 1992.

[7] N. Dyn. Subdivision schemes in CAGD. In William A. Light, editor, Advances in Numerical Analysis Vol. II, pages 36-104. Oxford University Press, 1992.

[8] N. Dyn. Analysis of convergence and smoothness by the formalism of Laurent polynomials. In Armin Iske, Ewald Quak, and Michael S. Floater, editors, Tutorials on multiresolution in geometric modelling, pages 51-68. Springer-Verlag, Berlin, 2002.

[9] N. Dyn, M. S. Floater, and K. Hormann. A $C^{2}$ four-point subdivision scheme with fourth order accuracy and its extensions. In Mathematical Methods for Curves and Surfaces: Tromsø 2004. Nashboro Press, Brentwood, 2005.

[10] N. Dyn, J. A. Gregory, and D. Levin. A four-point interpolatory subdivision scheme for curve design. Comput. Aided Geom. Design, 4:257-268, 1987. 
[11] N. Dyn and D. Levin. Analysis of asymptotically equivalent binary subdivision schemes. J. Math. Anal. Appl., 193:594-621, 1995.

[12] N. Dyn and D. Levin. Subdivision schemes in geometric modelling. Acta Numerica, pages 73-144, 2002.

[13] A. Karger and J. Novák. Space kinematics and Lie groups. Gordon and Breach, 1985.

[14] J. M. Lane and R. F. Riesenfeld. A theoretical development for the computer generation and display of piecewise polynomial surfaces. IEEE Trans. Pattern Anal. Mach. Intell. PAMI, 1:35-46, 1980.

[15] J. Nash. The imbedding problem for Riemannian manifolds. Annals of Math., 63:20-63, 1956.

[16] L. Noakes. Riemannian quadratics. In Alain Le Méhauté, Christopher Rabut, and Larry L. Schumaker, editors, Curves and Surfaces with Applications in CAGD, volume 1, pages 319-328. Vanderbilt University Press, Nashville and London, 1997.

[17] L. Noakes. Non-linear corner cutting. Adv. Comp. Math, 8:165-177, 1998.

[18] L. Noakes. Accelerations of Riemannian quadratics. Proc. Amer. Math. Soc., 127:1827$1836,1999$.

[19] A. L. Onishchik and E. B. Vinberg. Lie Groups and Algebraic Groups. Springer Verlag, Berlin, 1990.

[20] P. Oswald. Smoothness of nonlinear median-interpolation subdivision. Adv. Comput. Math., 20:401-423, 2004.

[21] G. Taubin. Estimating the tensor of curvature of a surface from a polyhedral approximation. In Proc. Int. Conf. Computer Vision, pages 902-907, 1995.

[22] J. Wallner and N. Dyn. Convergence and $C^{1}$ analysis of subdivision schemes on manifolds by proximity. Comput. Aided Geom. Des., 22:593-622, 2005.

[23] J. Wallner and H. Pottmann. Intrinsic subdivision with smooth limits for graphics and animation. ACM Trans. Graphics, 25(2);356-374, 2006.

[24] J. Warren and H. Weimer. Subdivision Methods for Geometric Design: A Constructive Approach. Morgan Kaufmann Series in Computer Graphics. Morgan Kaufmann, San Francisco, 2001.

[25] G. Xie and T. P.-Y. Yu. Smoothness analysis of nonlinear subdivision schemes of homogeneous and affine invariant type. Constructive Approximation, 22(2):219-254, 2005. 